Draft VERSION OCTOBER 18, 2018

Preprint typeset using LATEX style emulateapj v. 08/22/09

\title{
CORONAL LINES AND DUST FORMATION IN SN 2005ip: NOT THE BRIGHTEST, BUT THE HOTTEST TYPE IIn SUPERNOVA
}

\author{
Nathan Smith, Jeffrey M. Silverman, Ryan Chornock, Alexei V. Filippenko, Xiaofeng Wang, Weidong Li, \\ Mohan Ganeshalingam, Ryan J. Foley ${ }^{1,2}$, Jacob Rex, \& Thea N. Steele \\ Department of Astronomy, University of California, Berkeley, CA 94720-3411; nathans@astro.berkeley.edu \\ Draft version October 18, 2018
}

\begin{abstract}
We present optical photometry and spectroscopy of SN 2005ip for the first 3 yr after discovery, showing an underlying Type II-L supernova (SN) interacting with a steady wind to yield an unusual Type IIn spectrum. For the first $\sim 160 \mathrm{~d}$, it had a fast linear decline from a modest peak absolute magnitude of about -17.4 (unfiltered), followed by a plateau at roughly -14.8 for more than $2 \mathrm{yr}$. Initially having a normal broad-lined spectrum superposed with sparse narrow lines from the photoionized circumstellar medium (CSM), it quickly developed signs of strong CSM interaction with a spectrum similar to that of SN 1988Z. As the underlying SN II-L faded, SN 2005ip exhibited a rich high-ionization spectrum with a dense forest of narrow coronal lines, unprecedented among SNe but reminiscent of some active galactic nuclei. The line-profile evolution of SN 2005ip confirms that dust formation caused its recently reported infrared excess, but these lines reveal that it is the first $\mathrm{SN}$ to show clear evidence for dust in both the fast SN ejecta and the slower post-shock gas. SN 2005ip's complex spectrum confirms the origin of the strange blue continuum in SN 2006jc, which also had post-shock dust formation. We suggest that SN 2005ip's late-time plateau and coronal spectrum result from rejuvenated CSM interaction between a sustained fast shock and a clumpy stellar wind, where $\mathrm{X}$-rays escape through the optically thin interclump regions to heat the pre-shock CSM to coronal temperatures.
\end{abstract}

Subject headings: circumstellar matter - stars: mass loss - stars: winds, outflows - supernovae: individual (SN 2005ip)

\section{INTRODUCTION}

Core-collapse supernovae ( $\mathrm{SNe}$ ) show a variety of spectral properties (see Filippenko 1997 for a review) based primarily on the amount of mass shed by the progenitor, causing the outer layers of the star to be stripped to different chemical layers at the time of its explosion. In the Type IIn subclass, substantial mass stripping has occurred recently, leaving dense hydrogen gas in the circumstellar medium (CSM) into which the SN blast wave propagates. In this interaction, the blast wave is decelerated and the CSM is illuminated, giving rise to relatively narrow emission lines from the post-shock gas and the unshocked CSM.

The Type IIn subclass shows particularly wide diversity in both luminosity and spectral features, depending on density, speed, and how long before explosion the H-rich material was shed by the star. SNe IIn can be among the most luminous SNe observed if the CSM is very dense, as in the case of SN 2006tf (Smith et al. 2008b) - but they can also be among the faintest $\mathrm{SNe}$ observed as in the case of the so-called "supernova impostors" and related objects, which are not yet clearly understood (e.g., Van Dyk et al. 2005; Thompson et al. 2008). In order for the CSM interaction luminosity to compete with the main peak of the SN (arising from the diffusion of radioactive decay luminosity and shockdeposited energy in the SN ejecta), the CSM must be dense. At lower densities where the conversion of shock

\footnotetext{
${ }^{1}$ Present address: Harvard-Smithsonian Center for Astrophysics, 60 Garden St., Cambridge, MA 02138.

${ }^{2}$ Clay Fellow.
}

energy into visual light is less efficient, signs of weaker CSM interaction might become more prominent in the spectrum once the underlying SN fades; indeed, here we suggest that SN 2005ip was an example of the latter.

SN 2005ip was discovered (Boles 2005) on 2005 Nov. 5.163 (UT dates are used throughout this paper), located 2". $8 \mathrm{E}$ and $14.22 \mathrm{~N}$ of the center its host Scd galaxy NGC $2906(\sim 2.1 \mathrm{kpc}$ in projection on the sky). Fox et al. (2008) show images of SN 2005ip in its host galaxy. With an apparent redshift of $z=0.00714$ (de Vaucouleurs et al. 1991), NGC 2906 is located at a distance of roughly $29.7 \mathrm{Mpc}$ (adopting $H_{0}=72 \mathrm{~km} \mathrm{~s}^{-1} \mathrm{Mpc}^{-1}$ ). Modjaz et al. (2005) reported that in a spectrum obtained $\sim 1 \mathrm{~d}$ after discovery, SN 2005ip appeared to be a normal Type II event a few weeks after explosion, with a blue continuum and broad absorption features indicating a SN-ejecta expansion speed of roughly $15,400 \mathrm{~km} \mathrm{~s}^{-1}$. Recently, Fox et al. (2008) have shown that SN 2005ip had near-infrared (IR) excess emission from hot dust, and suggested that grains formed in the post-shock region, analogous to SN 2006jc (Smith et al. 2008a).

We present spectra showing that SN 2005ip also had narrow $\mathrm{H} \alpha$ emission indicative of a Type IIn classification, even on day 1, plus an unusually rich forest of narrow coronal emission lines that dominate the spectrum at later times. Immler \& Pooley (2007) reported a high X-ray luminosity at late times, implying a level of CSM interaction comparable to that of other SNe IIn like SN 1988Z. We present a brief discussion of the remarkable light curve and focus on the spectral evolution of SN 2005ip, interpreting it as the result of rejuvenated late-time CSM interaction in a steady wind with rela- 


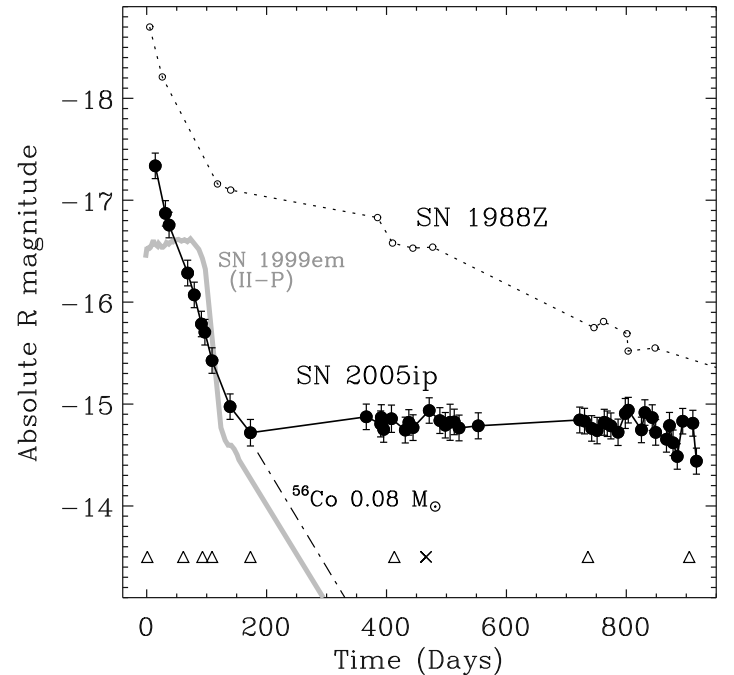

FIG. 1.- The unfiltered (approximately $R$-band) absolute magnitude light curve of SN 2005ip measured by KAIT, compared to that of SN 1988Z (from Turatto et al. 1993). The absolute magnitude of SN 2005ip is shown for a distance of $29.72 \mathrm{Mpc}$ and an extinction of $A_{R}=0.126 \mathrm{mag}$ (see text). The small triangles at the bottom mark dates for which we secured spectra of SN 2005ip, and the "X" marks the date (day 466) for which Immler \& Pooley (2007) observed its X-ray emission. The gray curve shows a normal SN II-P (SN 1999em) from our database for comparison, and the dot-dash line is ${ }^{56} \mathrm{Co}$ decay luminosity for $0.08 \mathrm{M}_{\odot}$ of ${ }^{56} \mathrm{Ni}$.

tively poor efficiency in converting shock energy to visual light. We confirm the suggestion by Fox et al. (2008) that dust formed, but our analysis of line profiles reveals that dust formed in both the post-shock shell and the fast SN ejecta at different times.

\section{OBSERVATIONS}

We monitored SN 2005ip photometrically using unfiltered images obtained with the Katzman Automatic Imaging Telescope (KAIT; Filippenko et al. 2001; Filippenko 2005) at Lick Observatory. Its host galaxy, NGC 2906, is included in the sample galaxies for the Lick Observatory Supernova Search with KAIT. As part of this search, unfiltered images of NGC 2906 have been obtained in recent years, but the field was behind the Sun before the discovery, so KAIT did not yield a useful non-detection limit before explosion. These data also constitute the follow-up photometry for SN 2005ip.

Flat fielding and bias subtraction were processed automatically. Galaxy subtraction and differential photometry were done using the KAIT pipeline (Ganeshalingam et al., in prep.), although background host-galaxy emission is faint around SN 2005ip. The unfiltered KAIT photometry is approximately equivalent to $R$-band photometry (Li et al. 2003), and the resulting unfiltered magnitudes are listed in Table 1 . The photometry is calibrated with the red magnitudes of stars in the USNO B1 catalog (Monet et al. 2003), and the uncertainty is the errors in photometry and calibration added in quadrature (but dominated by the scatter in the calibration). Adopting $m-M=32.365 \mathrm{mag}$ and $A_{R}=0.126 \mathrm{mag}$ for $E(B-V)=0.047 \mathrm{mag}$ (Schlegel et al. 1998), the absolute $\sim R$-band magnitude light curve is shown in Figure 1 , where we take day zero to be $\mathrm{JD}=2,453,679.66$, the discovery date 2005 Nov. 5 (Boles 2005).

We also obtained visual-wavelength spectra of
TABLE 1

Photometry of SN 2005IP

\begin{tabular}{ccc}
\hline JD & mag. & $\sigma$ \\
\hline 2453694.05 & 15.15 & 0.13 \\
2453711.03 & 15.62 & 0.13 \\
2453716.96 & 15.73 & 0.13 \\
2453747.91 & 16.20 & 0.13 \\
2453758.93 & 16.42 & 0.13 \\
2453770.91 & 16.70 & 0.13 \\
2453776.82 & 16.79 & 0.13 \\
2453788.84 & 17.06 & 0.13 \\
2453818.76 & 17.52 & 0.13 \\
2453852.70 & 17.77 & 0.13 \\
2454046.05 & 17.62 & 0.13 \\
2454070.04 & 17.69 & 0.13 \\
2454071.06 & 17.62 & 0.13 \\
2454074.97 & 17.74 & 0.13 \\
2454087.99 & 17.63 & 0.13 \\
2454110.93 & 17.75 & 0.13 \\
2454116.93 & 17.67 & 0.13 \\
2454123.88 & 17.72 & 0.13 \\
2454150.90 & 17.55 & 0.13 \\
2454168.80 & 17.65 & 0.13 \\
2454177.78 & 17.70 & 0.13 \\
2454185.71 & 17.70 & 0.18 \\
2454192.75 & 17.67 & 0.13 \\
2454200.74 & 17.76 & 0.13 \\
2454232.69 & 17.70 & 0.13 \\
2454402.02 & 17.65 & 0.13 \\
2454410.07 & 17.66 & 0.13 \\
2454422.07 & 17.73 & 0.13 \\
2454431.00 & 17.75 & 0.13 \\
2454443.04 & 17.67 & 0.13 \\
2454447.98 & 17.68 & 0.13 \\
2454454.06 & 17.71 & 0.13 \\
2454466.02 & 17.77 & 0.13 \\
2454478.00 & 17.58 & 0.15 \\
2454482.93 & 17.55 & 0.13 \\
2454504.89 & 17.74 & 0.13 \\
2454510.87 & 17.57 & 0.13 \\
2454522.91 & 17.62 & 0.13 \\
2454528.78 & 17.77 & 0.13 \\
2454546.79 & 17.84 & 0.13 \\
2454551.73 & 17.70 & 0.13 \\
2454557.77 & 17.87 & 0.13 \\
2454564.75 & 18.01 & 0.13 \\
2454573.72 & 17.66 & 0.13 \\
2454590.69 & 17.68 & 0.13 \\
2454596.68 & 18.05 & 0.13 \\
& & \\
\hline NoTE. - & KAIT & unfil- \\
tered photometry; roughly $R$ \\
band. & & \\
& &
\end{tabular}

TABLE 2

SPECTROSCOPY OF SN 2005IP

\begin{tabular}{cclccc}
\hline \hline UT Date & Day $^{\mathrm{a}}$ & Inst. & $\lambda / \Delta \lambda$ & Airmass & Exp. (s) \\
\hline 2005 Nov. 06.65 & 1 & DEIMOS & 2500 & 1.07 & 100 \\
2006 Jan. 05.40 & 61 & Kast & 600 & 1.18 & 1200 \\
2006 Feb. 06.40 & 93 & Kast & 700 & 1.19 & 1800 \\
2006 Feb. 22.36 & 109 & Kast & 600 & 1.18 & 1500 \\
2006 Apr. 27.26 & 173 & LRIS & 1100 & 1.03 & 504 \\
2006 Dec. 23.56 & 413 & DEIMOS & 2500 & 1.03 & 500 \\
2007 Nov. 11.63 & 736 & LRIS & 1500 & 1.10 & 600 \\
2008 Apr. 28.26 & 905 & LRIS & 1500 & 1.03 & 600 \\
& & & & & \\
\hline
\end{tabular}

a Days after discovery.

b Kast, Lick 3-m; LRIS, Keck I; DEIMOS, Keck II. 
SN 2005ip on eight separate dates using the Kast double spectrograph (Miller \& Stone 1993) on the 3-m Shane telescope at Lick Observatory, and using the Low Resolution Imaging Spectrometer (LRIS; Oke et al. 1995) and the Deep Imaging Multi-Object Spectrograph (DEIMOS; Faber et al. 2003) at Keck Observatory (see Table 2). Except when the object was at low airmass, the long slit (generally of width $\sim 2^{\prime \prime}$ at Lick and $\sim 0.7-1^{\prime \prime}$ at Keck) was oriented along the parallactic angle (Filippenko 1982) to avoid differential light losses due to atmospheric dispersion. Standard spectral data reduction was performed for all epochs (e.g., Foley et al. 2003), including a careful subtraction of background galaxy light by subtracting the spectrum on either side of the SN. Examples of the resulting spectra are plotted in Figure 2 after having been corrected for $E(B-V)=0.047 \mathrm{mag}$.

There may be additional reddening and extinction local to SN 2005ip, but the best way to correct for it is debatable. The day 1 spectrum shows local Na I absorption that may indicate additional reddening of as much as $E(B-V)=0.3 \mathrm{mag}$ (Munari \& Zwitter 1997), or $A_{R}=0.75 \mathrm{mag}$ assuming a standard value of $R=3.1$. However, this narrow $\mathrm{Na}$ I absorption feature is not clearly detected in later spectra, and most importantly, is absent in the day 413 DEIMOS spectrum with the same resolving power as on day 1 (although there is an extra emission feature that develops on the red wing of He I $\lambda 5876$ on day 413, which complicates the interpretation even further). Since we see clear evidence for this additional extinction only on day 1 and do not know precisely how $A_{R}$ varies with time, we have not corrected the light curve in Fig. 1. If present, the way that this additional reddening of as much as $E(B-V)=0.3 \mathrm{mag}$ on day 1 would affect our analysis below is as follows: (1) the peak absolute magnitude could have been about $0.75 \mathrm{mag}$ brighter than we quote below, (2) the effective temperature we derive from a blackbody approximation of the continuum on day 1 would be hotter, about $10^{4}$ $\mathrm{K}$ instead of $7300 \mathrm{~K}$, (3) the initial photospheric radius would be smaller by about a factor of 2 , and (4) the corresponding constraints to the date of explosion would be $4-5 \mathrm{~d}$ before day 1 , rather than $8-10 \mathrm{~d}(\S 3.2)$. None of these corrections would alter our interpretation about the overall nature of SN 2005ip or its progenitor.

\section{RESULTS}

\subsection{The Unusual Light Curve of SN 2005ip}

The light curve of SN 2005ip (Fig. 1) shows a rapid decline from its peak magnitude at the time of discovery, with the early decline at $\sim 0.019 \mathrm{mag} \mathrm{d}^{-1}$ reminiscent of SNe II-L, perhaps discovered a few weeks after explosion (in that case, its peak magnitude may have been more luminous; e.g., Doggett \& Branch 1985; Arnett 1996). If it was discovered near its peak, then its luminosity was typical of average Type II-P or II-L events (Fig. 1), lacking the enhanced luminosity that is common in strongly interacting SNe IIn like SN 2006tf (Smith et al. 2008b) or SN 1988Z (Turatto et al. 1993; Artexaga et al. 1999). Attributing the luminosity of the declining tail around day 150 to the radioactive decay of ${ }^{56} \mathrm{Co}$ would indicate a maximum initial ${ }^{56} \mathrm{Ni}$ mass of $\sim 0.08 \mathrm{M}_{\odot}$. (The explosion date is not known, but if it is, say, 3 weeks prior to discovery, then the implied ${ }^{56} \mathrm{Ni}$ mass could be $\sim 0.1 \mathrm{M}_{\odot}$. .)
From $\sim 160$ days after discovery until the present time, however, the red light curve of SN 2005ip exhibits unusual behavior: its decline hit a "floor" and remained essentially constant at an absolute magnitude of roughly -14.8 (Fig. 1). Slowly declining SNe are rare, but to have a SN stay at constant visual luminosity for several years after explosion is unprecedented. SN 1988Z had qualitatively similar behavior with a similar kink in its light curve, although SN $1988 \mathrm{Z}$ was more luminous than SN 2005ip and it had a slow decline rather than a true plateau. As we show below, this late-time plateau of SN 2005ip is contributed in large part by strong $\mathrm{H} \alpha$ emission plus a number of other narrow lines over a flat and weak red continuum.

\subsection{The Unusual Spectra of SN 2005ip}

Figure2 shows the spectral evolution of SN 2005ip. At first glance, our earliest spectrum on day 1 is not very peculiar; SN 2005ip has a normal Type II spectrum with a smooth blue continuum and broad P Cygni profiles for He I $\lambda 5876$ and $\mathrm{H} \alpha$, indicating outflow speeds of almost $19,000 \mathrm{~km} \mathrm{~s}^{-1}$ from the blueshifted trough of $\mathrm{H} \alpha$. The continuum shape in the dereddened spectrum on day 1 can be matched with a blackbody at $7300 \mathrm{~K}( \pm 100 \mathrm{~K}$; although see caveats in $\S 2$ regarding the effect of possible additional reddening). Its luminosity at that early time indicates a characteristic blackbody radius of $1.4 \times 10^{15}$ $\mathrm{cm}$. This radius, combined with the observed expansion speed, constrains the date of explosion to be at least 8 $10 \mathrm{~d}$ before discovery.

However, a closer look at the day 1 spectrum also reveals a sparse series of narrow emission lines superposed on the spectrum (background light from the host galaxy has been subtracted), so SN 2005ip was a Type IIn event even at the earliest times. The narrow lines observed in our day 1 DEIMOS spectrum (5000-10,000 $\AA$ ) are $\mathrm{H} \alpha$, He I $\lambda \lambda 5876,6680,7065,[\mathrm{Fe}$ VII] $\lambda \lambda 5720,6086,[\mathrm{Fe} \mathrm{x}]$ $\lambda 6375$, and [Fe XI] $\lambda 7891$ ([O III] $\lambda 5007$ may have also been present but was near the blue edge of the detector where the wavelength calibration is questionable). These high-ionization lines are commonly seen in active galactic nuclei (AGNs) (e.g., Filippenko \& Sargent 1989), novae (e.g., Williams et al. 1991), and the solar corona (e.g., Wagner \& House 1968), but are rare in $\mathrm{SNe}$ (but see Gordon 1972).

Between days 1 and 150, SN 2005ip faded more rapidly than the ${ }^{56}$ Co decay rate (Fig. 11). During that time, it developed a prominent broad $\mathrm{H} \alpha$ line from the SN ejecta, with a width near zero intensity of $\pm 16,000 \mathrm{~km} \mathrm{~s}^{-1}$, while other lines of intermediate width from the post-shock gas and very narrow lines from the pre-shock CSM became more prominent. The overall character of the spectrum during this phase - as well as the width and strength of $\mathrm{H} \alpha$ - was very similar to that of SN $1988 \mathrm{Z}$ (Filippenko 1991; Turatto et al. 1993; Stathakis \& Sadler 1991). In fact, Figure 2 shows that the only substantial difference between the day 61 spectrum of SN 2005ip and the day 193 spectrum of SN 1988 Z is that the broad O I $\lambda 8446$ feature from the fast ejecta is stronger in SN 1988Z (the O I line strengthens later in SN 2005ip).

By the time the underlying SN II-L fades away (after day 160) and SN 2005ip begins its long constantluminosity phase, its spectrum has undergone a remarkable transformation. Narrow coronal lines now domi- 


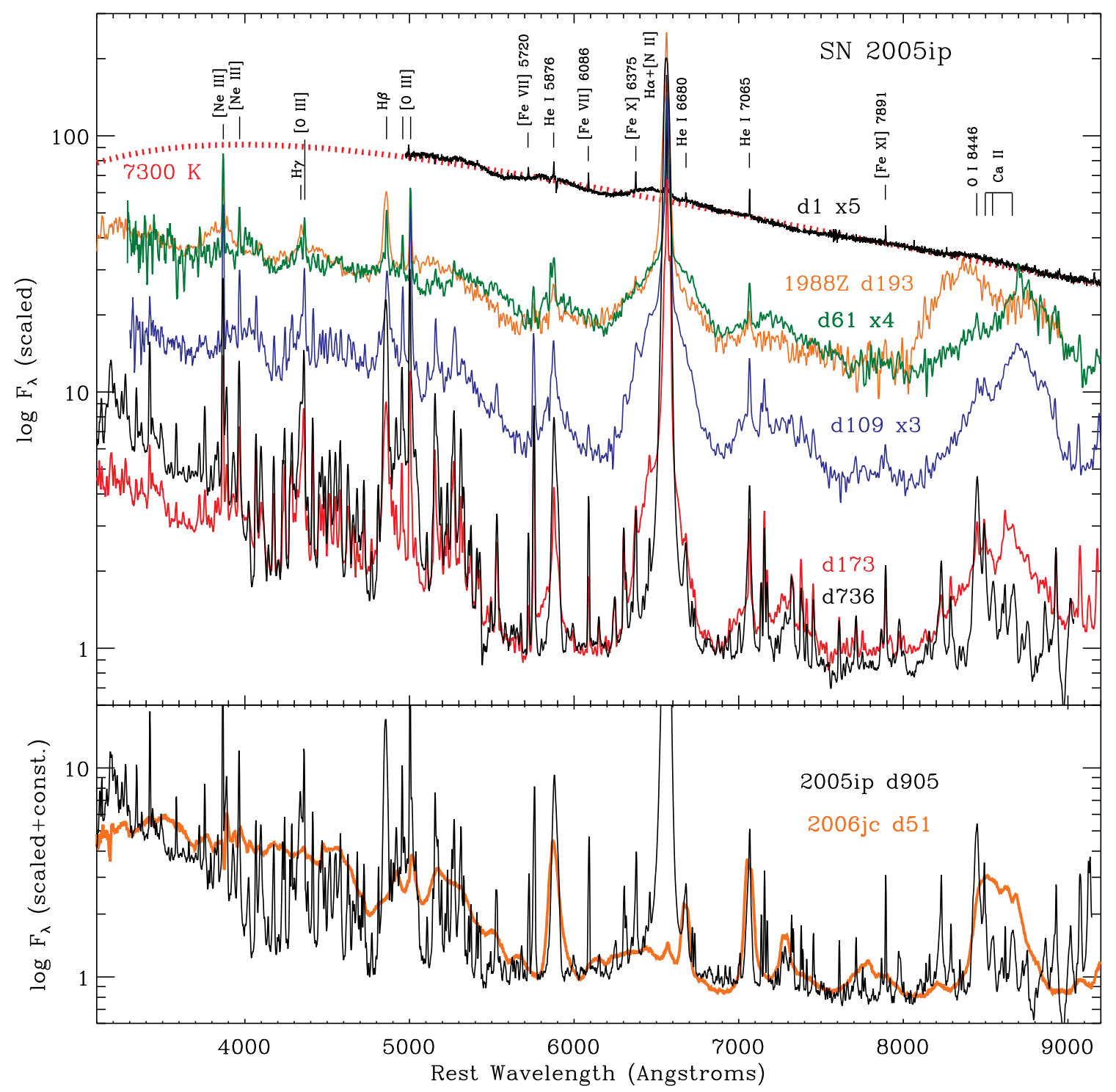

Fig. 2.- Top Panel: Spectra of SN 2005ip on days 1 (black), 61 (green), 109 (blue), 173 (red), and 763 (black). For comparison, we also show the day 193 spectrum of SN 1988Z (orange) from our spectral database. These have been corrected for reddening as noted in the text, and the days 1, 61, and 109 spectra have been scaled as noted in the figure for better visibility. Bottom Panel: Same as above but showing the day 905 spectrum of SN 2005ip (black) compared to the day 51 spectrum of SN 2006jc (orange) from Smith et al. (2008a). SN 2006jc is H-depleted and the lines are broader, but the unusual continuum shape is similar to SN 2005ip.

nate the appearance of the spectrum, along with some intermediate-width lines from post-shock regions (He I and Balmer lines). Examples of the spectra dominated by these narrow lines (days 173 and 736) are shown in Figure 2, These coronal lines will be discussed in more detail below in $\S 3.4$.

\subsection{The Blue Pseudo-Continuum and SN 2006jc}

At early times, the shape of SN 2005ip's visual continuum can be approximated by that of a $\sim 7300 \mathrm{~K}$ blackbody spectrum, typical of SNe II (Fig. 2). As the SN fades, however, narrow emission lines begin to dominate the spectrum and the underlying continuum takes on a different character. At red wavelengths $(\lambda>5500 \AA)$, the continuum at later times is too flat to be matched by any single blackbody shape, while shortward of $5500 \AA$, the continuum rises too steeply to be fit by any blackbody. This pronounced blue bump is apparent in the day 109 spectrum, it is well developed by day 173 , and it persists at least until our last available spectrum on day 905 (Fig. 2).

It is striking how well the continuum shape of SN 2005ip mimics the blue pseudo-continuum and flat red continuum of SN 2006jc (Foley et al. 2007; Smith et al. 2008a), and a comparison of the two is shown in the bottom panel of Figure 2. With the exception of $\mathrm{H} \alpha$, the two match quite well (note that the strong and broad Ca II triplet in SN 2006jc comes from the underlying SN ejecta and is present in earlier spectra of SN 2005ip). This close match implies two things, as follows.

First, if SN 2005ip were H-deficient and had higher speeds in the CSM (broader lines), its spectrum might closely resemble that of SN 2006jc (SN 2005la may be an intermediate case; Pastorello et al. 2008). Since this blue feature dominates the visual broad-band luminosity of SN 2005ip for $\sim 3 \mathrm{yr}$, it must arise as a result of lu- 


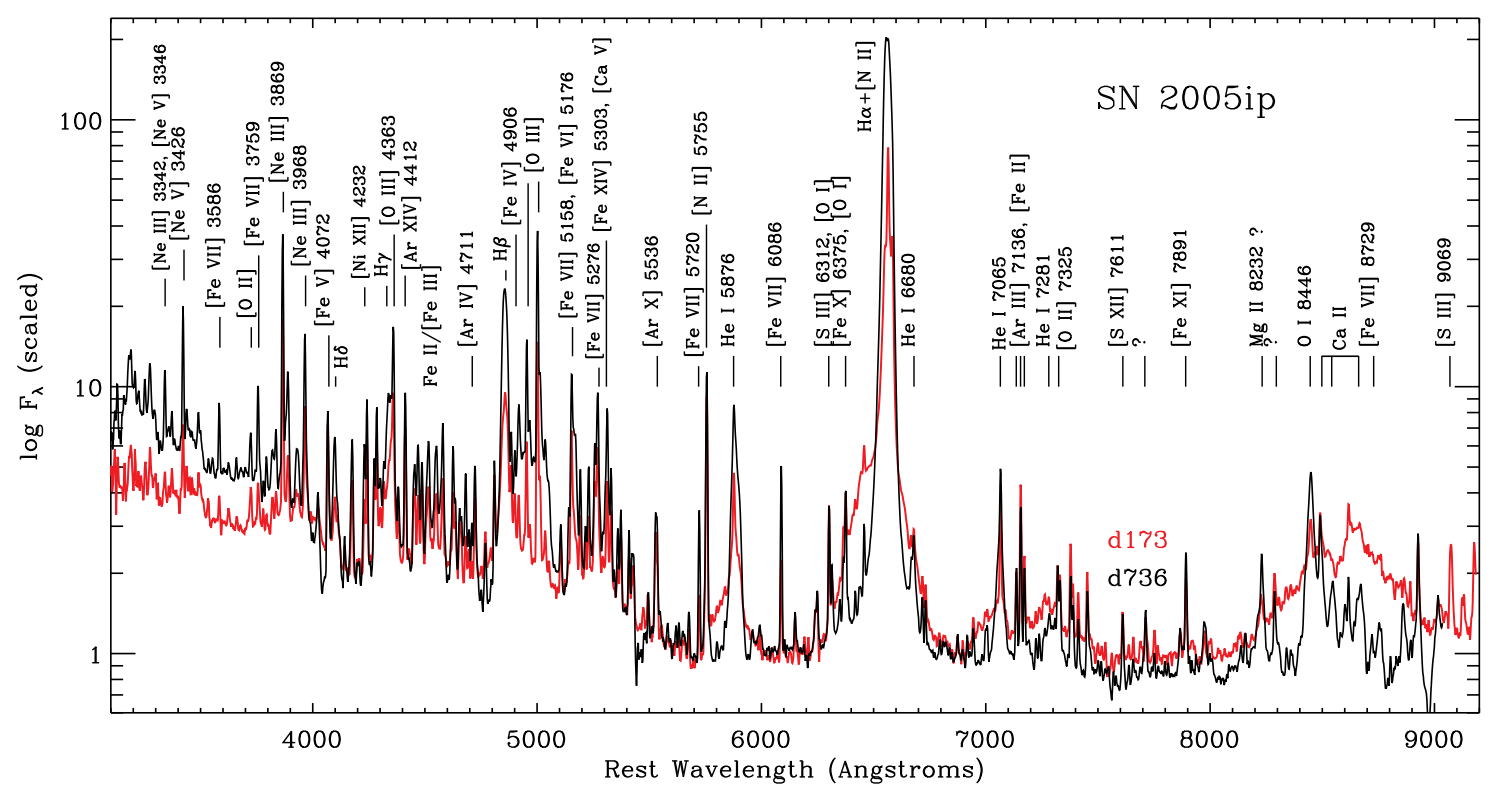

FIG. 3.- Spectra of SN 2005ip on days 173 (red) and 763 (black), with a number of probable line identifications.

minosity generated by ongoing CSM interaction and not radiation from the underlying SN ejecta. This was unclear in SN 2006jc, because the SN faded more quickly (Foley et al. 2007). Also, the fact that the blue feature is seen in both SNe 2005ip and 2006jc means that it is not the result of an increased burden of cooling on fluorescent lines caused by the lack of $\mathrm{H}$ opacity, because SN 2005ip is not H-deficient.

Second, because the CSM lines in SN 2005ip are narrower, we see that the blue pseudo-continuum can in fact be decomposed into a forest of narrow emission lines. Some regions of the spectrum with a particularly dense clustering of narrow lines in SN 2005ip, such as 4000 $4700 \AA$, correspond to a bumpy blue "continuum" with an overall flux distribution similar to that of SN 2006jc. This overlap therefore supports our earlier hypothesis (Foley et al. 2007; Smith et al. 2008a) that the strong blue "continuum" in SN 2006jc was fluorescence from a number of blended emission lines. We emphasize that the individual lines are seen in SN 2005ip only by virtue of their formation in its slow $150-200 \mathrm{~km} \mathrm{~s}^{-1}$ CSM, whereas the progenitor of SN 2006jc was a Wolf-Rayet (WR) star with wind speeds of order $2000 \mathrm{~km} \mathrm{~s}^{-1}$. With faster CSM speeds, the forest of narrow lines in SN 2005ip would have blended together in a pseudo-continuum as well.

The fact that the blue emission arises unambiguously in the CSM in SN 2005ip has interesting implications for SN 2006jc. Foley et al. (2007) first noted that in SN 2006jc, only He I lines that were superposed on the blue pseudo-continuum had $\mathrm{P}$ Cygni absorption associated with them. By analogy with SN 2005ip, one might conclude that the P Cygni absorption arose in the cooler outer wind of SN 2006jc's progenitor, while the blue pseudo continuum arises from fluorescence in the inner wind or post-shock gas. The lack of P Cygni absorption then requires that the source of the red continuum was cooler gas than that producing the He I lines, probably the recombining SN ejecta.

A similar (although less pronounced) blue pseudocontinuum was also seen in SN $1988 \mathrm{Z}$ (see Fig. 2), as noted by Turatto et al. (1993). Turatto et al. pointed out the anomalously blue color of SN 1988 Z due to a peculiar rise at 3500-5700 $\AA$, as in SNe 2005ip and 2006jc, and correctly speculated that it may be due to the overlap of a huge number of emission lines. Since both SNe $1988 \mathrm{Z}$ and 2005ip showed coronal emission lines and the blue continuum, one might infer a direct link between the two processes. SN 2006jc, however, did not exhibit the forbidden coronal lines for reasons that are unclear.

Another important point to note when comparing the continua of SNe 2005ip and 2006jc is that while both showed the flat red continuum, they behaved differently with time. For a limited time, SN 2006jc developed a strong red/IR excess indicative of hot dust at $\sim 1600 \mathrm{~K}$ (Smith et al. 2008a); combined with the blue continuum, this gave the visual-wavelength spectrum of SN 2006jc a "U"-like shape, which eventually disappeared. SN 2005ip did not exhibit this behavior, indicating that its dust was not hot enough or not luminous enough to dominate the red continuum. If the newly condensing grains in SN 2005ip are significantly cooler than the $\sim 1600 \mathrm{~K}$ grains in SN 2006jc, this may argue in favor of silicate grains in SN 2005ip instead of the C-rich grains that probably formed in SN 2006jc (see Smith et al. 2008a).

\subsection{Narrow Lines from the Highly Ionized CSM}

The many narrow lines that dominate the late-time spectrum of SN 2005ip are noteworthy for their high ionization level and wide range in ionization. At late-times, many more of these high-excitation lines have appeared, beyond the few visible on day 1 (Fig. 2). The narrow species seen prominently on day 1 were $[\mathrm{Fe}$ VII], [Fe X], [Fe XI], $\mathrm{H} \alpha$, and $\mathrm{He}$ I. In addition to these, late-time spectra show [O III], [Ne III], [Ne V], [S III], [S XII], [Ca V], [Ar III], [Ar IV], [Ar X], [Ar XIV], [Fe IV], [Fe V], [Fe VI], [Fe XIV], [Ni XII], and others. Identifications for several lines are given in Figures 3 and 4 .

As noted earlier, these high-ionization lines are seen in novae, AGNs, and the solar corona, but they are unusual in SNe. In fact, no other SN has exhibited such 


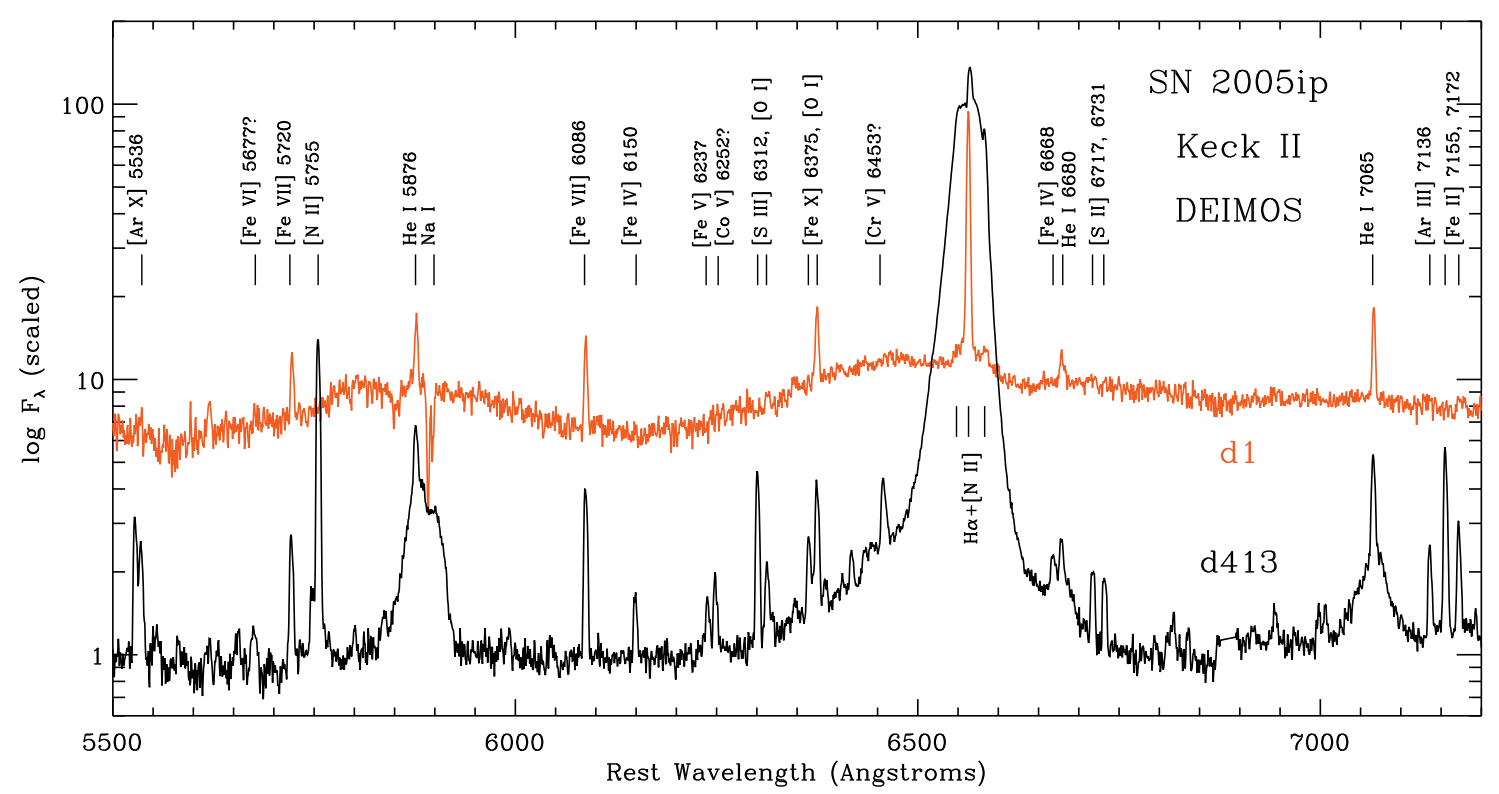

FIG. 4.- A selected wavelength range around $\mathrm{H} \alpha$ as seen in spectra obtained with high spectral resolution using the DEIMOS spectrograph on Keck II, on days 1 (gray; orange in the online edition) and 413 (black). Coronal lines have only narrow components, whereas H $\alpha$ and $\mathrm{He}$ I also show intermediate-width components from post-shock gas at the later epoch, and broad H $\alpha$ emission is seen from the underlying fast SN ejecta.

a rich spectrum of narrow coronal lines. A few of the stronger lines were seen in the spectrum of SN $1988 \mathrm{Z}$ (Turatto et al. 1993), reinforcing the overall similarities between SNe 2005ip and 1988Z that we have mentioned above. However, the number of lines, their strength, the range of ionization, and the highest-ionization level seen are all higher in SN 2005ip. Fransson et al. (2002) presented a detailed analysis of narrow coronal lines in the SN IIn $1995 \mathrm{~N}$, showing many of the same coronal lines we detect in SN 2005ip. Again, though, the coronal lines in SN $1995 \mathrm{~N}$ were not as prominent as in our late-time spectra of SN 2005ip, and a comparison of the evolution of coronal lines in the two SNe is not possible because it is thought that SN $1995 \mathrm{~N}$ was first discovered $\sim 10$ months after explosion. In general, though, the physical conditions in SN 1995N's CSM derived by Fransson et al. (2002) and the origin of its coronal lines are similar to those from our analysis of SN 2005ip.

SN 1987A also showed faint emission from a subset of these coronal features, including [Fe XIV] $\lambda 5303$, in intermediate-width lines from dense shock-heated gas (Fransson \& Gröningsson 2007; Gröningsson et al. 2006, 2008). This emission appeared 10-20 yr after explosion, once the forward shock began plowing into its dense ring nebula. Some of these features may also have been present in SN 1986J (Leibundgut et al. 1991), in the earliest spectra of SN 1993J (Garnavich \& Ann 1994), and were seen as weak narrow lines in the SN IIn 1997eg (Hoffman et al. 2008). While these relatively weak coronal lines have been seen in a few other SNe, the way that they dominate the spectral morphology of SN 2005ip is unprecedented among known SNe. Note that Figures 2 and 3 are plotted on a logarithmic scale, so the narrow lines are quite strong compared to the continuum.

Emission from many lines with a wide range of ionization - especially the highest ionization — implies high temperature gas compared to that of most SNe. The highest ionization species we detect is the relatively weak [Fe XIV] $\lambda 5303$ emission line (at most epochs, it is severely blended with [Ca V] $\lambda 5309)$. The presence of [Fe XIV] $\lambda 5303$ requires that a substantial fraction of the CSM has electron temperatures up to $2 \times 10^{6} \mathrm{~K}$. Most other lines suggest substantial amounts of gas at several $10^{5} \mathrm{~K}$ and below. Since this coronal emission dominates the overall spectrum to a greater degree than in any other SN during its late CSM-interaction phase, we conclude that the bulk of the emitting gas in SN 2005ip is hotter than in any other known SN IIn.

\subsection{Origin of the Coronal Lines}

One normally associates such wide ranges of ionization and temperature with the post-shock cooling zone, but all of SN 2005ip's coronal lines are very narrow, indicating that they cannot come from the post-shock gas - they must arise in the pre-shock CSM. This is demonstrated most clearly in Figure 4, which shows our two epochs of high-resolution Keck/DEIMOS spectra on days 1 and 413 after discovery. In Figure 4, one can see broad emission from the SN ejecta in the broad component of $\mathrm{H} \alpha$, intermediate-width components emitted by post-shock gas in both $\mathrm{H} \alpha$ and He I lines, and only narrow emission components for all other lines.

The profiles of the narrow lines are unresolved in all of our Lick/Kast and Keck/LRIS spectra, but they appear to be resolved in our high-resolution DEIMOS spectrum, with varying FWHMs of 120 (unresolved) to $240 \mathrm{~km} \mathrm{~s}^{-1}$. Lines this narrow are not formed in the SN ejecta or in the post-shock gas like the intermediate-width $(\sim 1000$ $3000 \mathrm{~km} \mathrm{~s}^{-1}$ ) lines usually seen in SNe IIn (see, e.g., Smith et al. 2008b; Chugai \& Danziger 1994).

Since many of the ions giving rise to the narrow lines have ionization potentials corresponding to soft X-ray energies, and since their narrow widths cannot be produced in the post-shock gas, we conclude that the unusual spectrum of coronal lines in SN 2005ip arises from pre-shock ionization of the CSM by X-rays emitted by the shocked 


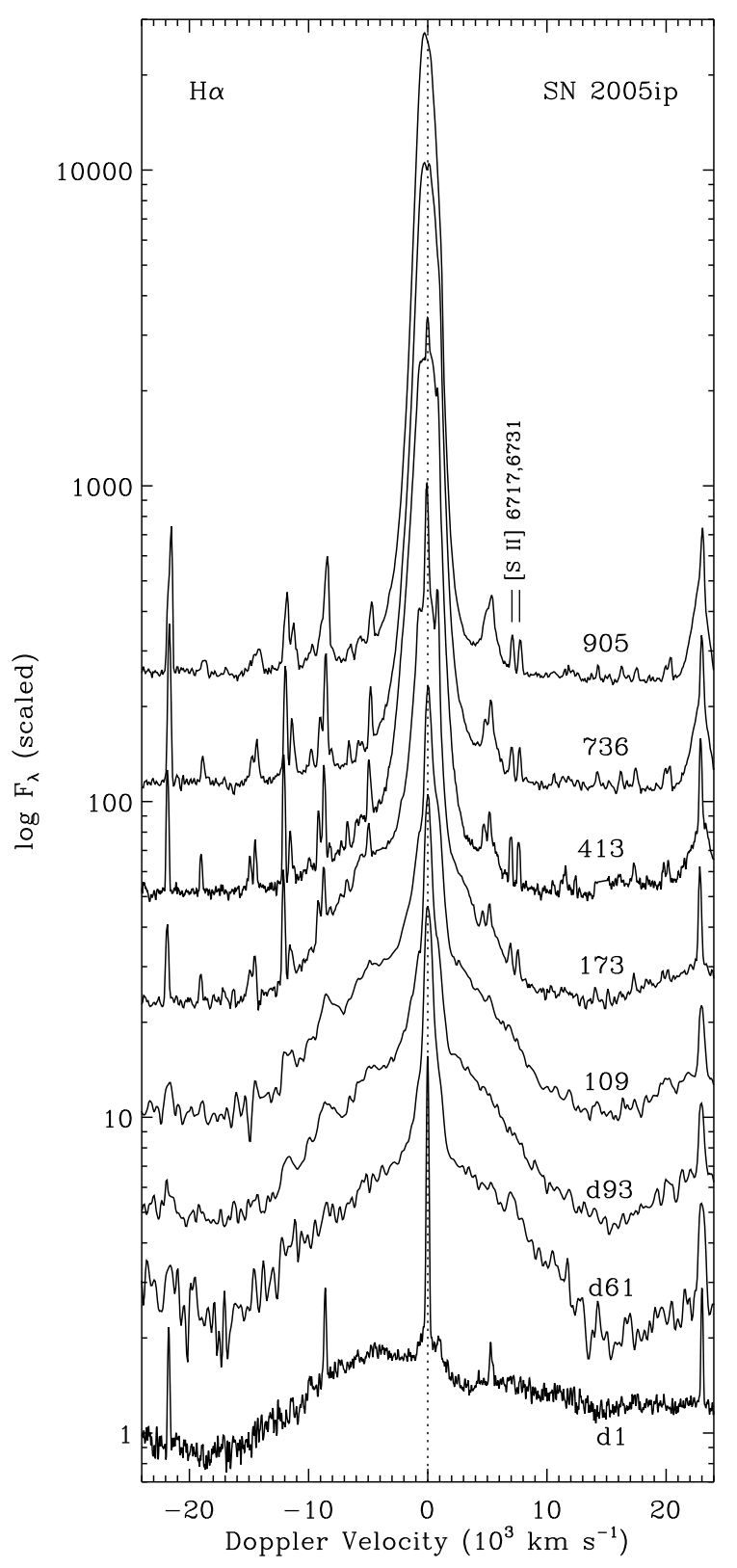

FIG. 5.- $\mathrm{H} \alpha$ line-profile evolution in SN 2005ip. Changes can be seen in the relative strength of broad components $( \pm 16,000 \mathrm{~km}$ $\left.\mathrm{s}^{-1}\right)$, as well as intermediate-width $\left(\sim 10^{3} \mathrm{~km} \mathrm{~s}^{-1}\right)$ and narrow components. Days 61-109 do not have sufficiently high dispersion to resolve the narrow components from the intermediate-width components.

gas. Potential sources of X-rays are the initial shock breakout or sustained X-ray emission from ongoing CSM interaction (or both), and these will be considered in more detail below (§3.7) where we discuss the observed line intensities.

\subsection{Line-Profile Evolution}

$\mathbf{H} \alpha$ and $\mathbf{H} \beta$ : The time evolution of the $\mathrm{H} \alpha$ profile is shown in Figures 5 and [6. Ignoring the superposed coronal and other narrow lines, we find that the underlying $\mathrm{H} \alpha$ line profile shape, strength, and evolution with time are remarkably similar to those of the $\mathrm{H} \alpha$ line in SN $1988 Z$ (Stathakis \& Sadler 1991; Turatto et al. 1993).

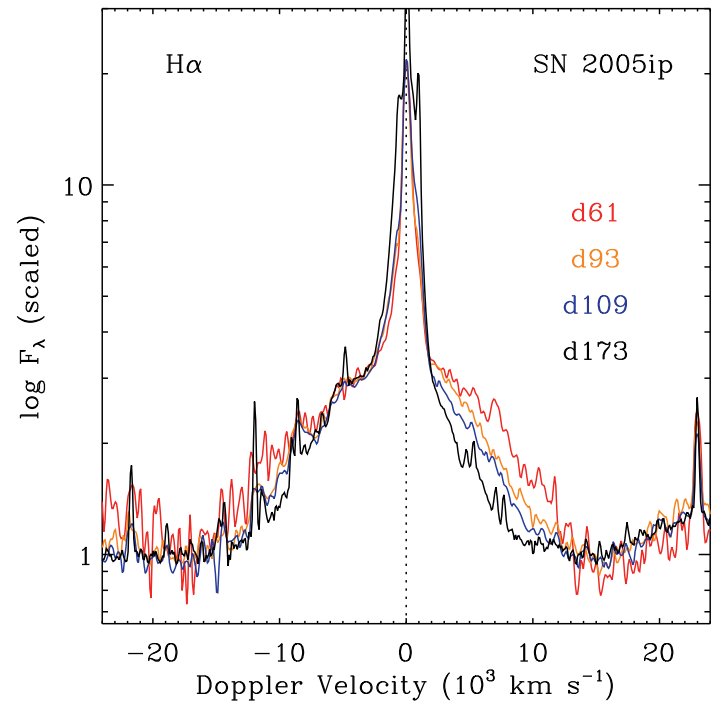

Fig. 6.- Same as Fig. 5 but showing only days 61,93 , 109, and 173 during the main luminosity decline of SN 2005ip, scaled and superposed on one another. The red side of the broad $\mathrm{H} \alpha$ profile indicates dust formation within the expanding fast ejecta.

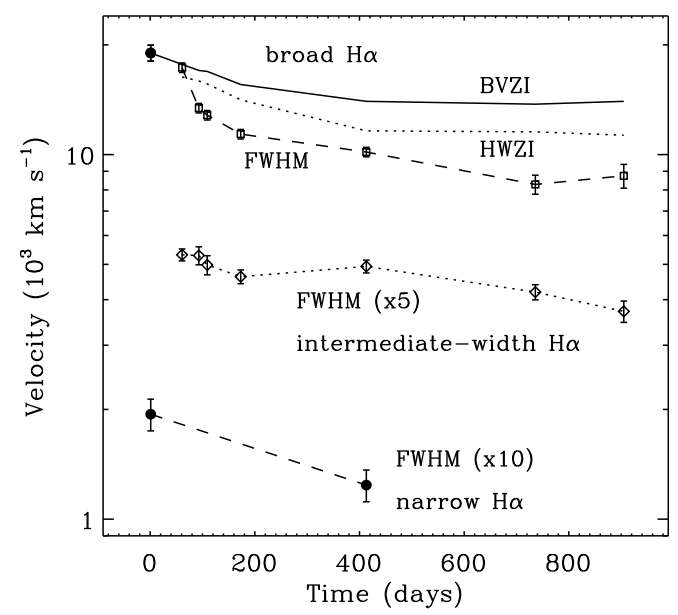

FIG. 7.- Measured velocities for $\mathrm{H} \alpha$ as a function of time. For the broad component (top), we show the velocity of the P Cygni absorption trough for day 1 , followed by the blue velocity at zero intensity (BVZI), the half-width at zero intensity (HWZI), and the full-width at half maximum (FWHM) for the remaining epochs. FWHM values were measured with a Gaussian fit, but the fits were quite poor for the last two epochs because the broad component is much fainter than the intermediate-width component and is also crowded with several narrow coronal lines. The FWHM of the intermediate-width component is multiplied by a factor of 5 for display here, and the FWHM of the narrow component (the narrow-line profiles are only resolved in our DEIMOS spectra on days 1 and 413) is multiplied by 10 .

At most epochs, the $\mathrm{H} \alpha$ profile of SN 2005ip can be separated into three main components: (1) a broad component from the SN ejecta (either unshocked or having just passed the reverse shock) within typically $\pm 16,000 \mathrm{~km} \mathrm{~s}^{-1}$, decreasing with time (see Fig. 7), (2) an intermediate-width component at $\pm 1100 \mathrm{~km} \mathrm{~s}^{-1}$, and (3) a narrow component from the unshocked CSM at \pm 100 $200 \mathrm{~km} \mathrm{~s}^{-1}$. In the day 1 spectrum, only components (1) and (3) can be seen; the intermediate-width component is absent. The intermediate-width component steadily increases its strength with time as the broad compo- 


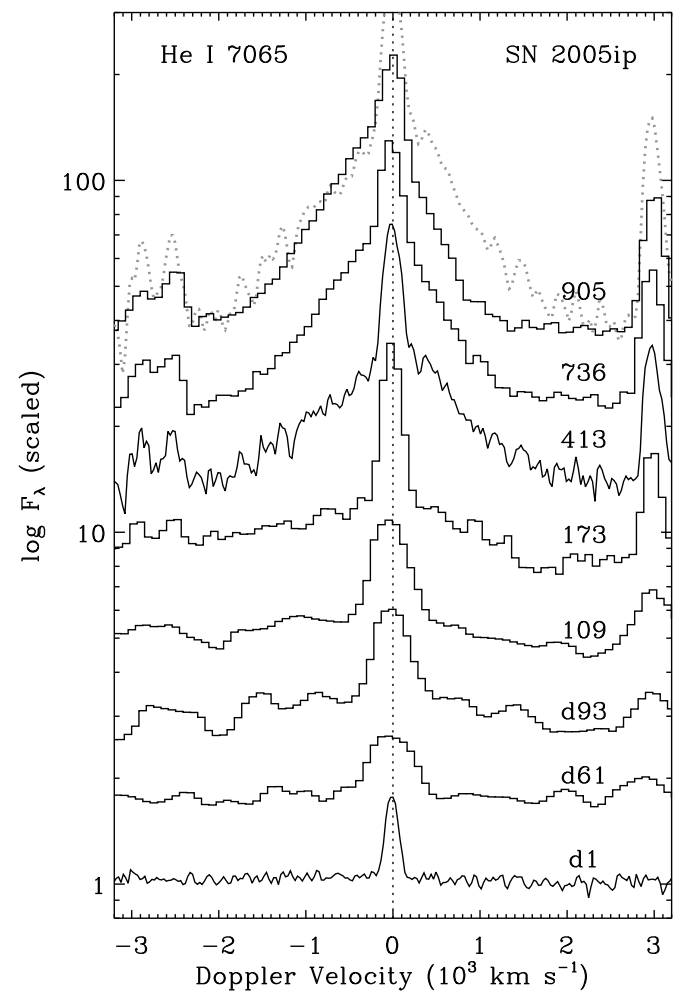

FIG. 8.- Line-profile evolution of He I $\lambda 7065$ in SN 2005ip. Changes can be seen in the relative strength of the narrow component, as well as an increase with time in the intermediate-width (few $10^{3} \mathrm{~km} \mathrm{~s}^{-1}$ ) component. Except for days 1 and 413, the narrow components are unresolved. The gray dotted curve is the day 413 profile with a relatively symmetric intermediate-width component, plotted over the day 905 spectrum to demonstrate evidence suggesting new dust formation at late times.

nent fades, such that the intermediate component comes to dominate the $\mathrm{H} \alpha$ flux at late times on the plateau of the light curve (note that Fig. [5 is logarithmic, so that at late times the broad component is actually quite weak). At late times the broad component has faded and slowed such that it appears to merge with the wings of the intermediate-width component and the two become difficult to distinguish (electron scattering may also contribute to the line wings here). Also, at the latest epochs, the narrow CSM component of $\mathrm{H} \alpha$ disappears.

During the main luminosity decline of SN 2005ip in the first $170 \mathrm{~d}$ after discovery, the broad component of $\mathrm{H} \alpha$ develops an increasingly asymmetric profile. As shown in Figure 6, the red wing of the line fades more quickly than its blue wing, producing a systematic blueward shift of the line center. This behavior can be attributed to dust grains that increasingly and selectively block out the far side of the SN ejecta as more dust forms. Since we also note the possibility of dust formation in the swept-up shell from He I line profiles (see below; also see Fox et al. 2008), SN 2005ip may be the first clear case where dust is seen to form in both the SN ejecta and the swept-up post-shock shell.

The measured velocities for the three components of $\mathrm{H} \alpha$ are shown in Figure 7 and listed in Table 3. On day 1, the broad component exhibits a smooth P Cygni profile; we show the speed of the blueshifted absorption trough as a representative SN ejecta velocity, but the intrinsic FWHM without blueshifted absorption could be greater

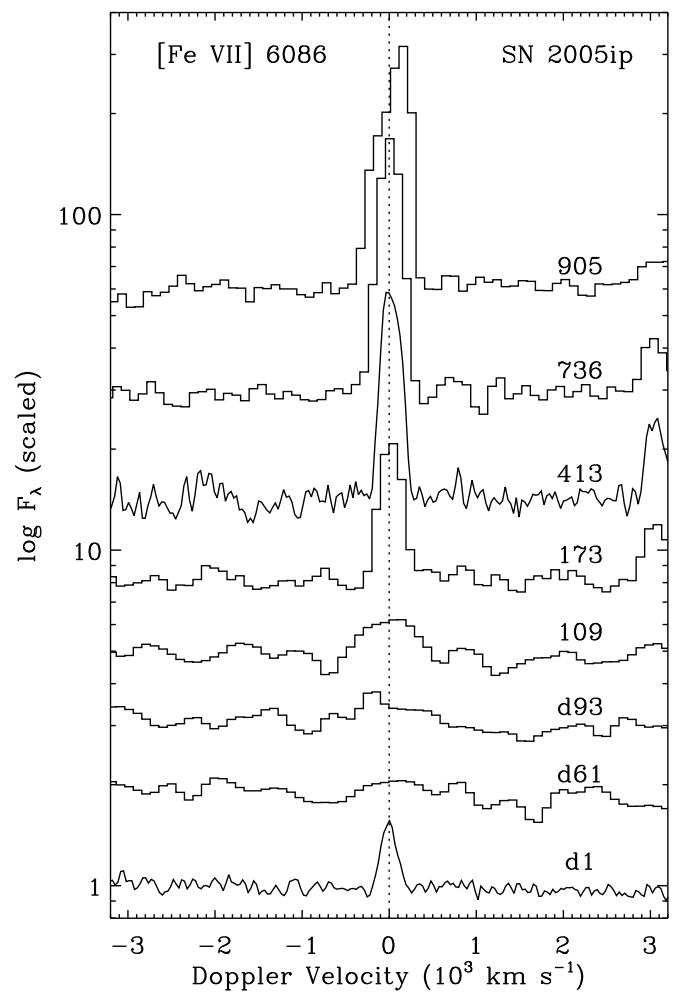

FIG. 9.- Line-profile evolution of the coronal [Fe VII] $\lambda 6086$ line in SN 2005ip. Only the narrow component from the CSM is detected. The only two epochs with sufficiently high dispersion to resolve the narrow-component profiles are days 1 and 413, so the apparent increase in line width at late times may be a resolution effect.

than this. For the remaining epochs, no clear P Cygni absorption feature is seen in the broad component. Since the broad line becomes asymmetric and is blended with the very strong intermediate-width component, FWHM values derived from Gaussian fits depend on the parameters one chooses for the fit. As an independent check, we also show the blue velocity at zero intensity (BVZI) and the half-width at zero intensity (HWZI) for comparison. The difference between the BVZI and HWZI reflects the line's asymmetry, as noted above. One can see that the BVZI does not decline as fast or as much, indicating that fast SN ejecta with speeds of $\sim 15,000 \mathrm{~km} \mathrm{~s}^{-1}$ are still crossing the reverse shock at late times, even though the FWHM suggests lower speeds.

The FWHM of the intermediate-width component from the post-shock CSM gas drops from $\sim 1100 \mathrm{~km} \mathrm{~s}^{-1}$ at early times to $\sim 800 \mathrm{~km} \mathrm{~s}^{-1}$ at late times. Qualitatively, this deceleration of the cold dense shell is expected from models of CSM interaction (e.g., Chugai \& Danziger 1994). The uncertainty in the intermediate-width component's FWHM value is dominated by the unknown contribution of [N II] $\lambda 6583$. The uncertain contribution of $[\mathrm{N}$ II] $\lambda 6583$, in turn, makes it problematic to investigate possible effects of post-shock dust formation on the red wing of the intermediate-width component of $\mathrm{H} \alpha$. To this end, we use He I lines instead (see below).

The narrow component also shows a reduction in the observed width with time, from $190 \mathrm{~km} \mathrm{~s}^{-1}$ on day 1 to $124 \mathrm{~km} \mathrm{~s}^{-1}$ on day 413 (at the same spectral resolution). This may suggest that the progenitor's wind speed increased in the decades immediately before the SN, or 
TABLE 3

H $\alpha$ Component Velocities, Total Flux, Equivalent Width, and Balmer Decrement

\begin{tabular}{|c|c|c|c|c|c|c|c|c|c|}
\hline Epoch & $\begin{array}{l}\text { Br. P Cyg } \\
(\mathrm{km} \mathrm{s}-1)\end{array}$ & $\begin{array}{l}\text { Br. BVZI } \\
\left(\mathrm{km} \mathrm{s}^{-1}\right)\end{array}$ & $\begin{array}{l}\text { Br. HWZI } \\
\left(\mathrm{km} \mathrm{s}^{-1}\right)\end{array}$ & $\begin{array}{l}\text { Br. FWHM } \\
\left(\mathrm{km} \mathrm{s}^{-1}\right)\end{array}$ & $\begin{array}{l}\text { Int. FWHM } \\
\left(\mathrm{km} \mathrm{s}^{-1}\right)\end{array}$ & $\begin{array}{l}\text { Nar. FWHM } \\
\left(\mathrm{km} \mathrm{s}^{-1}\right)\end{array}$ & $\begin{array}{c}\text { Flux } \\
\left(\operatorname{erg~s} \mathrm{s}^{-1} \mathrm{~cm}^{2}\right)\end{array}$ & $\begin{array}{l}\mathrm{EW} \\
(\AA)\end{array}$ & $\mathrm{H} \alpha / \mathrm{H} \beta$ \\
\hline Day 1 & $18500(900)$ & $\ldots$ & $\ldots$ & [16300] & $\ldots$ & 194(19) & $1220 \times 10^{-16}$ & 49.5 & $\cdots$ \\
\hline Day 93 & $\ldots$ & 17000 & 15920 & $13400(400)$ & $1060(300)$ & $\cdots$ & $2870 \times 10^{-16}$ & 1240 & 10.6 \\
\hline Day 109 & $\ldots$ & 16900 & 15620 & $12800(380)$ & $1000(300)$ & $\cdots$ & $2400 \times 10^{-16}$ & 1550 & 10.8 \\
\hline Day 173 & $\cdots$ & 15600 & 14130 & $11400(340)$ & $930(200)$ & $\ldots$ & $1790 \times 10^{-16}$ & 3070 & 9.8 \\
\hline Day 413 & $\cdots$ & 14000 & 11620 & $10200(300)$ & $990(200)$ & $124(12)$ & $2650 \times 10^{-16}$ & 5760 & 11.6 \\
\hline Day 736 & $\cdots$ & 13800 & 11550 & $8300(500)$ & $840(200)$ & $\ldots$ & $3250 \times 10^{-16}$ & 8880 & 14.3 \\
\hline Day 905 & $\cdots$ & 14000 & 11300 & $8800(660)$ & $740(250)$ & $\cdots$ & $3340 \times 10^{-16}$ & 9320 & 24.7 \\
\hline
\end{tabular}

Note. - BVZI and HWZI uncertainties are roughly $\pm 500 \mathrm{~km} \mathrm{~s}^{-1}$. The FWHM for the broad (Br.) emission component on day 1 is in brackets because it is an underestimate of the true FWHM due to strong P Cygni absorption. Uncertainties for the H $\alpha$ total flux and equivalent width are roughly $\pm 5 \%$.

that the luminosity of the SN itself accelerated CSM material at small radii but had less influence on the more distant CSM. This may also explain why different narrow lines have different widths, if they trace different radial zones of ionization and density in the wind.

He I lines: We take the strong triplet line He I $\lambda 7065$ as representative of the behavior of He I line profiles arising in the post-shock shell. He I $\lambda 5876$, although strong, is problematic because its red wing is affected by $\mathrm{Na}$ I D. The evolution of the He I $\lambda 7065$ line profile is shown in Figure 8. The relative strength of the narrow component from the unshocked CSM stays nearly constant during the main fading of the SN. An intermediate-width component from post-shock gas $\left( \pm 1800 \mathrm{~km} \mathrm{~s}^{-1}\right)$ is absent at early times during the main light-curve peak; it first becomes conspicuous at day 173 and increases in its relative strength thereafter, coming to dominate the total He I $\lambda 7065$ flux at late times. At late epochs, this intermediate-width component becomes asymmetric, with a deficit of emission in its red wing. In Figure 8 , the asymmetric day 905 profile of He I $\lambda 7065$ is compared to the more symmetric profile on day 413 (dashed gray). The relative fading of the red sides of the intermediatewidth He I line is reminiscent of that seen in SN 2006jc (Smith et al. 2008a), and it implies dust formation in the post-shock shell as we will discuss later in $\S 4.1$.

Coronal lines: To investigate the evolution of lineprofile shapes for the high-ionization coronal lines, we consider the observed behavior of [Fe VII] $\lambda 6086$ (Fig. 9]), because it is relatively strong and not blended with other lines. At the earliest epoch (d1), the line profile is the same as for He I $\lambda 7065$, indicating a pure line from the undisturbed CSM. As time progresses, however, [Fe VII] $\lambda 6086$ evolves very differently from He I $\lambda 7065$. During the main luminosity decline in the first $\sim 100 \mathrm{~d}$, [Fe VII] $\lambda 6086$ weakens considerably with respect to the fading continuum. It increases in relative strength thereafter, but it never develops the intermediate-width components that are seen in He I lines. Instead, it remains as a narrow line emitted exclusively by the highly ionized pre-shock CSM. (Note that although the width of the line appears to increase from day 413 to days 736 and 905 in Fig. 9 , this is a resolution effect attributed to the lower dispersion of the days 736 and 905 spectra; see Table 2.)

\subsection{Line Intensities}

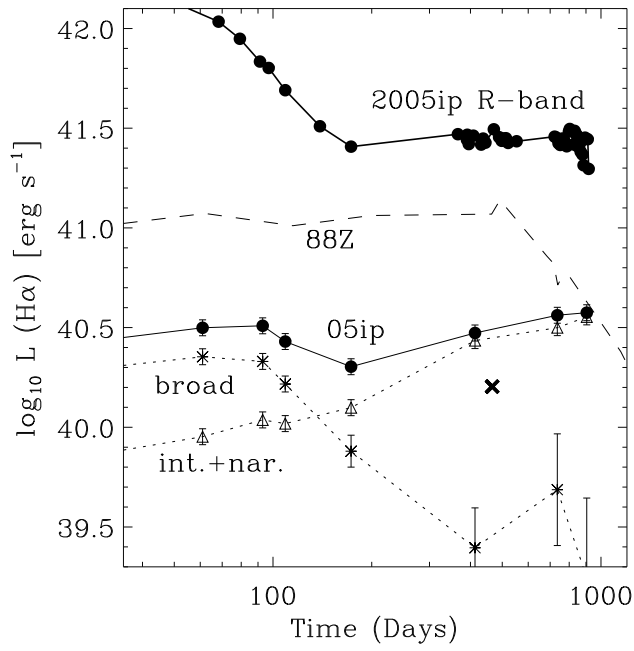

FIG. 10.- The total H $\alpha$ line luminosity of SN 2005ip, compared to that of SN 1988Z (Artexaga et al. 1999). We also show the bolometric luminosity of SN 2005ip derived from the $R$-band photometry, as well as the X-ray luminosity (shown by the "X") measured by Immler \& Pooley (2007). Asterisks show the contribution of the broad component to the $\mathrm{H} \alpha$ line of SN 2005ip, which decays with time, and triangles show the sum of the intermediate-width and narrow components, increasing with time.

$\mathbf{H} \alpha$ : Figure 10 shows how the $\mathrm{H} \alpha$ luminosity of SN 2005ip changes with time, compared to its underlying continuum and also with $\mathrm{H} \alpha$ in SN 1988Z. In both SNe, the total $\mathrm{H} \alpha$ luminosity remains roughly constant as the underlying SN fades, although at late times SN 1988Z's $\mathrm{H} \alpha$ luminosity begins to drop, whereas that of SN 2005ip remains constant and even rises slightly until the end of our observations. Despite this nearly constant luminosity, the line profile changes substantially (Fig. 5). Decomposing the total $\mathrm{H} \alpha$ luminosity into the broad component and the narrow plus intermediate-width components (Fig. 10) reveals two distinct phases. At early times during the main fading of the underlying SN II-L, the broad $\mathrm{H} \alpha$ dominates. As it fades with the SN, the intermediate and narrow components strengthen and come to dominate at late times. At the latest epochs, however, Figure [5] shows that the narrow component disappears and $\mathrm{H} \alpha$ is dominated by only the intermediate component.

This reflects the fact that as the SN ejecta expand and 


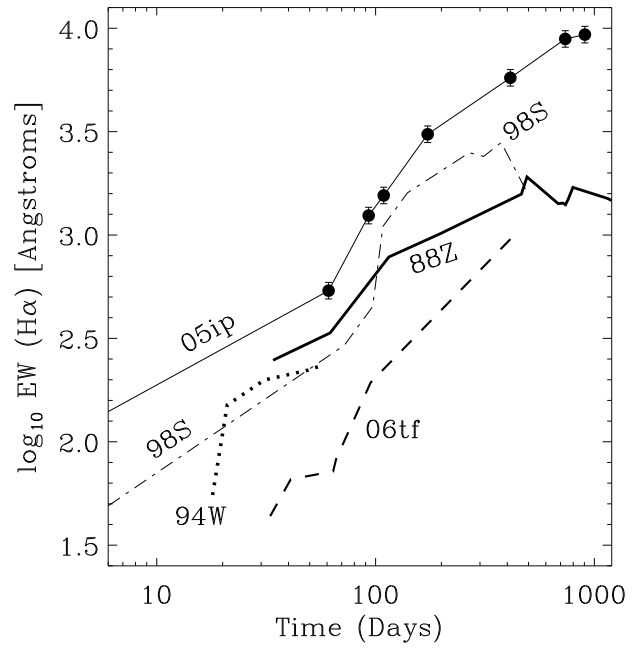

FIG. 11. - The total H $\alpha$ equivalent width of SN 2005ip, compared to those of SNe 1988Z, 1998S, 1994W, and 2006tf (see Fig. 13 of Smith et al. 2008b and references therein).

cool, more $\mathrm{H}$ atoms from the CSM are swept up into the post-shock shell and heated by X-rays from that ongoing CSM interaction, probably mainly from the reverse shock (Chevalier \& Fransson 1994). Note that the escaping Xray luminosity measured by Immler \& Pooley (2007) is only half the $\mathrm{H} \alpha$ luminosity at the same epoch (Fig.10), suggesting that a large fraction (at least half to $2 / 3$ ) of the intrinsic X-ray luminosity is absorbed by the sweptup shell, sustaining the optical luminosity, and indicating that $\mathrm{H} \alpha$ is an important coolant.

It is likely that the fraction of SN 2005ip's total X-ray luminosity absorbed by the post-shock shell is lower than that of most other SNe IIn (i.e., it has a lower efficiency in converting shock kinetic energy into light). This is based on the fact that a substantial fraction of the X-rays must escape the post-shock shell to reach the CSM and energize the coronal spectrum that is more prominent than in any other SN, and because SN 2005ip's observed X-ray luminosity is comparable to that of SN $1988 \mathrm{Z}$ even though the visual CSM interaction luminosity is less.

The lower efficiency of converting shock energy and Xrays into visual light may be connected to the reason why SN 2005ip has a greater $\mathrm{H} \alpha$ equivalent width than other SNe IIn (Fig. 11). Specifically, a lower effective optical depth in the swept-up shell could allow more X-rays to escape, and might also place a larger burden of cooling on $\mathrm{H} \alpha$ as opposed to the continuum. That lower effective optical depth might be due to either lower densities and a lower progenitor mass-loss rate, or perhaps a higher degree of clumping or asymmetry (see $\S 4.2$ and 4.3).

He I and Narrow/Coronal Lines: Figure 12 shows the temporal behavior of luminosities for a few representative narrow lines, while Table 4 lists the measured fluxes or upper limits for many narrow lines. A sparse sample of narrow coronal lines is seen on day 1. Some of these remain bright as the SN evolves, whereas others initially fade to non-detectability and then gradually return at late times. Examples of the former are [Fe VII] $\lambda 6086$, [O III] $\lambda 5007$, and He I $\lambda 7065$, while examples of the latter behavior are [Fe x] $\lambda 6375,[\mathrm{Fe} \mathrm{xI}] \lambda 7891$ and He I $\lambda 6680$. Most narrow lines that appear later, with

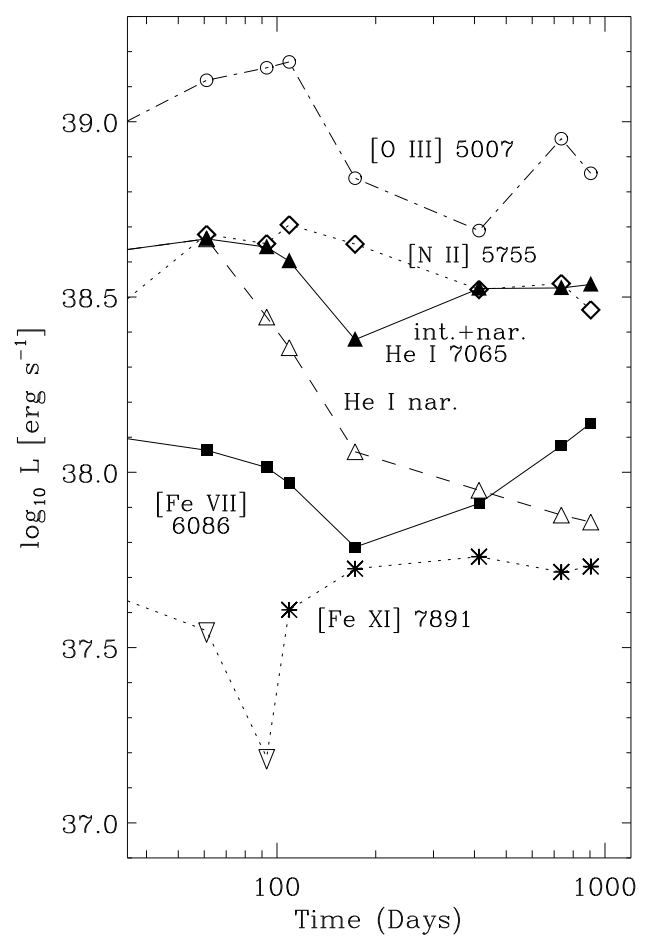

FIG. 12.- Same as Fig. 10 but comparing narrow-line luminosities of He I $\lambda 7065$ (both narrow [unfilled triangles] and intermediate-width [filled]), [O III] $\lambda 5007$ (circles), [N II] $\lambda 5755$ (diamonds), [Fe VII] $\lambda 6086$ (filled squares), and [Fe XI] $\lambda 7891$ (asterisks). We do not plot [Fe XIV] $\lambda 5303$ because it is blended with [Ca v] $\lambda 5309$.

both moderate and high ionization, have only upper limits on day 1 but then strengthen dramatically as time proceeds. This is particularly true for lines of relatively low ionization (e.g., [O I], [O II], [S II]).

The variety of behavior seen in SN 2005ip's spectrum can be quite complicated and perplexing. Consider the evolution of a few lines in particular: one sees very similar changes with time for the total flux in $\mathrm{H} \alpha$ (Fig. 10), He I $\lambda 7065$, [O III] $\lambda 5007$, and [Fe VII] $\lambda 6086$ (Fig. 12). All four lines show an initial "hump" in their light curves that has a broad peak just before day 100, followed by marked decline following the SN continuum luminosity to reach a minimum around day 200 , and then all four lines recover their strength at late times. In $\mathrm{H} \alpha$, the initial "hump" is emission from the broad component formed in the fast SN ejecta, which then fades as the intermediatewidth component takes over the flux in the line (Fig. 10). For He I, however, the initial hump is dominated by the narrow component which fades as the intermediate-width component comes to dominate (Fig. 12). Both [O III] and [Fe VII] are seen exclusively as narrow lines at all times, never developing an intermediate-width component even though their total intensity recovers at late times like $\mathrm{H} \alpha$ and He I. It is surprising that the total-flux evolution in all four lines is so similar when their different subcomponents arise in three different spatial zones within the SN - the fast ejecta, the post-shock gas, and the unshocked CSM, all with different ranges of density and temperature. We conjecture that the differing behavior in the subcomponents of these lines reflects different ways of processing the same energy source emerging mainly 
TABLE 4

Fluxes of Selected Narrow/Coronal Lines in SN 2005iP

\begin{tabular}{|c|c|c|c|c|c|c|c|c|c|}
\hline I.D. & $\begin{array}{c}\lambda \\
(\AA)\end{array}$ & $\begin{array}{l}\text { Flux d1 } \\
\left(10^{-16}\right)\end{array}$ & $\begin{array}{l}\text { Flux d61 } \\
\left(10^{-16}\right)\end{array}$ & $\begin{array}{l}\text { Flux d93 } \\
\left(10^{-16}\right)\end{array}$ & $\begin{array}{l}\text { Flux d109 } \\
\left(10^{-16}\right)\end{array}$ & $\begin{array}{l}\text { Flux d173 } \\
\quad\left(10^{-16}\right)\end{array}$ & $\begin{array}{l}\text { Flux d413 } \\
\quad\left(10^{-16}\right)\end{array}$ & $\begin{array}{l}\text { Flux d736 } \\
\quad\left(10^{-16}\right)\end{array}$ & $\begin{array}{l}\text { Flux d905 } \\
\quad\left(10^{-16}\right)\end{array}$ \\
\hline [Ne III],[Ne V] & 3342,46 & $\cdots$ & $\cdots$ & $35.3(6.67)$ & & $(2.86)$ & $\cdots$ & $15.3(1.81)$ & $13.6(4.21)$ \\
\hline$[\mathrm{Ne} v]^{2}$ & 3426 & $\cdots$ & $\cdots$ & $16.1(6.64)$ & $11.9(7.26)^{*}$ & $12.6(1.85)$ & $\cdots$ & $34.0(4.58)$ & $30.7(1.63)$ \\
\hline$[\mathrm{Fe}$ VII $]$ & 3586 & $\cdots$ & $\ldots$ & $(6.29)$ & $(5.25)$ & $5.10(1.50)$ & $\cdots$ & $10.3(1.44)$ & $8.45(1.07)$ \\
\hline$\left[\begin{array}{ll}\mathrm{O} & \mathrm{II}\end{array}\right]$ & 3727 & $\cdots$ & $45.5(12.1)$ & $9.45(2.31)$ & $8.35(2.31)$ & $6.94(1.60)$ & $\cdots$ & $7.34(1.20)$ & $5.69(0.94)$ \\
\hline$[\mathrm{Fe}$ VII $]$ & 3759 & $\cdots$ & $(16.2)$ & $12.9(4.37)$ & $(5.06)$ & $6.39(2.40)$ & $\cdots$ & 16.1(1.48) & $12.4(1.85)$ \\
\hline$[\mathrm{Ne}$ III] & 3869 & $\cdots$ & $153(13.9)$ & $105(4.81)$ & $112(7.22)$ & $60.0(5.26)$ & $\cdots$ & $81.9(9.42)^{*}$ & $62.2(5.84)^{*}$ \\
\hline$\left[\begin{array}{ll}\mathrm{Ne} & \mathrm{III}\end{array}\right]$ & 3968 & $\cdots$ & $89.9(20.4)$ & $38.6(5.21)$ & $33.9(9.21)^{*}$ & $30.7(7.03)^{*}$ & $\cdots$ & $29.0(1.89)^{*}$ & $14.4(1.68)^{*}$ \\
\hline$\left[\begin{array}{ll}\mathrm{Fe} & \mathrm{V}\end{array}\right]$ & 4072 & $\cdots$ & $(17.3)$ & $22.8(7.39)^{*}$ & $22.8(1.65)^{*}$ & $24.1(5.08) *$ & $\ldots$ & $18.8(2.76)^{*}$ & $11.7(2.15)$ \\
\hline $\mathrm{H} \delta$ & 4103 & $\cdots$ & $(1.29)$ & $(5.07)$ & $(4.49)$ & $5.33(1.47)^{*}$ & $\cdots$ & $13.0(1.25)^{*}$ & $19.7(3.21)$ \\
\hline$[\mathrm{Ni} \quad \mathrm{XII}]$ & 4232 & $\ldots$ & $(0.78)$ & $(5.16)$ & $6.36(2.41)^{*}$ & $7.93(2.54)^{*}$ & $\ldots$ & $15.1(5.87)^{*}$ & $11.0(3.99)^{*}$ \\
\hline $\mathrm{H} \gamma$ & 4340 & $\ldots$ & $(1.74)$ & $18.6(1.75)$ & $17.6(2.47)^{*}$ & $(3.10)$ & $\ldots$ & $52.8(14.3) *$ & $37.0(8.93)^{*}$ \\
\hline$\left[\begin{array}{ll}\mathrm{O} & \mathrm{III}\end{array}\right]$ & 4363 & $\cdots$ & $43.2(7.10)$ & $39.1(8.69)^{*}$ & $39.7(4.79)^{*}$ & $32.6(7.79)^{*}$ & $\ldots$ & $33.9(4.77) *$ & $25.0(5.81) *$ \\
\hline$[$ Ar XIV] & 4412 & $\cdots$ & (1.08) & $22.1(3.94)$ & $22.9(1.68)$ & $23.7(1.50)$ & $\ldots$ & $21.5(0.81)$ & $12.6(1.06)$ \\
\hline$\left[\begin{array}{ll}\text { Ar } & \text { IV }\end{array}\right]$ & 4711 & $\ldots$ & $(0.85)$ & (10.9) & (2.19) & (1.94) & $(0.39)$ & $2.98(0.65) *$ & $1.72(0.76)^{*}$ \\
\hline$[\mathrm{Ne}$ IV $]$ & 4725 & $\ldots$ & $10.9(2.11)$ & $(5.97)$ & $7.84(5.80)$ & $11.0(0.97)$ & $(0.36)$ & $11.7(0.67)^{*}$ & $5.32(0.83) *$ \\
\hline $\mathrm{H} \beta$ & 4861 & $\ldots$ & $66.7(10.8)$ & $26.8(3.81)^{*}$ & $21.7(4.65)^{*}$ & $11.5(2.20)^{*}$ & $45.8(3.87)^{*}$ & $190(15.8) *$ & $125(5.54)$ \\
\hline$\left[\begin{array}{ll}\mathrm{Fe} & \mathrm{IV}\end{array}\right]$ & 4906 & $(5.97)$ & (6.11) & $(7.58)$ & $5.37(0.74)$ & $5.28(0.63)$ & $4.06(0.91)$ & $3.39(0.64)^{*}$ & $2.87(1.51)^{*}$ \\
\hline He I & 4922 & $(2.79)$ & $(8.14)$ & (6.67) & $7.70(4.84)$ & $6.56(0.74)$ & $4.49(0.69)$ & $7.64(0.99)^{*}$ & $12.0(1.87)^{*}$ \\
\hline$\left[\begin{array}{ll}\mathrm{O} & \mathrm{III}\end{array}\right]$ & 4959 & $(6.35)$ & $36.3(3.06)$ & $40.0(3.18)$ & $31.5(5.34)$ & $15.1(2.22)$ & $13.6(1.79)$ & $23.6(2.81)^{*}$ & $14.6(3.23)^{*}$ \\
\hline$\left[\begin{array}{ll}\mathrm{O} & \mathrm{III}\end{array}\right]$ & 5007 & $16.2(3.04)$ & $117(32.8)$ & $127(11.3)$ & $132(33.6)$ & $61.5(3.63)^{*}$ & $43.6(4.68)$ & $79.7(11.1) *$ & $63.5(8.96)^{*}$ \\
\hline$[\mathrm{Fe}$ VII $]$ & 5158 & $(1.34)$ & $12.3(5.84)$ & $19.3(3.71)^{*}$ & $15.8(4.76)^{*}$ & $20.3(5.29)^{*}$ & $14.9(1.96)^{*}$ & $19.4(2.19)^{*}$ & $13.4(3.17)^{*}$ \\
\hline$[\mathrm{Fe} \quad \mathrm{VI}]$ & 5176 & $3.23(1.57)$ & $9.64(3.00)$ & $(5.09)$ & $(1.58)$ & $(1.94)$ & $(0.98)$ & $(2.84)$ & $3.10(0.85)^{*}$ \\
\hline$\left[\begin{array}{ll}\mathrm{Fe} & \mathrm{VII}\end{array}\right]$ & 5276 & $(0.76)$ & $19.6(7.47)^{*}$ & $23.3(2.40)^{*}$ & $43.4(5.04)^{*}$ & $25.6(2.65)^{*}$ & $13.1(1.13)^{*}$ & $29.0(3.69)^{*}$ & $16.1(2.29)^{*}$ \\
\hline$\left[\begin{array}{lll}\mathrm{Fe} & \mathrm{XIV}\end{array}\right],[\mathrm{Ca} \mathrm{V}]$ & 5303,09 & $11.9(3.66)$ & $7.70(4.85)$ & $13.6(4.39)^{*}$ & $(1.77)$ & $10.4(0.95)$ & $1.10(0.34)^{*}$ & $12.7(1.26)^{*}$ & $8.59(1.84)^{*}$ \\
\hline$\left[\begin{array}{lll}\mathrm{Fe} & \mathrm{II}\end{array}\right]$ & 5328 & $(1.55)$ & (7.94) & $(2.09)$ & $9.69(2.35)^{*}$ & $6.29(0.57)$ & $(0.29) *$ & $5.76(1.07)^{*}$ & $2.63(0.52)^{*}$ \\
\hline$\left[\begin{array}{ll}\text { Ar } & \mathrm{X}\end{array}\right]$ & 5536 & $(2.75)$ & $(6.03)$ & $7.06(0.85)^{*}$ & $\ldots *$ & $\ldots *$ & $4.72(0.71) *$ & $10.2(3.16)^{*}$ & $7.27(2.70)^{*}$ \\
\hline$\left[\begin{array}{ll}\mathrm{Fe} & \mathrm{VII}\end{array}\right]$ & 5720 & $12.5(2.30)$ & $17.4(5.52)^{*}$ & (3.33) & $3.43(0.37)^{*}$ & $3.15(0.33)$ & $4.38(0.55)$ & $7.28(0.20)$ & $7.13(2.17)$ \\
\hline$[\mathrm{N}$ II $]$ & 5755 & $(1.86)$ & $42.5(5.22)$ & $40.0(6.49)$ & $45.3(6.43)$ & $39.9(3.58)$ & $29.6(4.57)$ & $30.8(1.87)$ & $25.9(1.96)$ \\
\hline He I (Nar.) & 5876 & $17.3(2.29)$ & $83.9(7.66)^{*}$ & $38.4(2.79)^{*}$ & $17.9(2.22)^{*}$ & $11.1(0.91)^{*}$ & $8.01(0.87)^{*}$ & $12.1(2.13)^{*}$ & $15.8(1.25)^{*}$ \\
\hline He I (Tot) & 5876 & $17.3(2.29)$ & $83.9(7.66)^{*}$ & $54.3(3.64)^{*}$ & $49.8(5.34)^{*}$ & $57.3(3.91)^{*}$ & $76.24(3.56)^{*}$ & $84.47(4.32)^{*}$ & $91.0(2.8)^{*}$ \\
\hline$[\mathrm{Fe}$ VII $]$ & 6086 & $18.1(2.54)$ & $10.3(1.80)^{*}$ & $9.2(1.22)^{*}$ & $8.29(1.50)^{*}$ & $5.45(0.35)$ & $7.26(1.01)$ & $10.6(0.60)$ & $12.3(2.56)$ \\
\hline$\left[\begin{array}{ll}\mathrm{O} & \mathrm{I}\end{array}\right]$ & 6300 & $(1.71)$ & (7.09) & $11.7(1.64)^{*}$ & $6.27(1.06)^{*}$ & $9.00(0.80)$ & $8.11(0.98)$ & $6.92(0.57)^{*}$ & $5.83(1.59)^{*}$ \\
\hline$\left[\begin{array}{ll}\mathrm{S} & \mathrm{III}\end{array}\right]$ & 6312 & $(3.41)$ & $(7.65)$ & $\ldots *$ & $7.07(0.54)^{*}$ & $2.64(1.04)^{*}$ & $1.99(0.26)$ & $3.18(0.78) *$ & $2.64(1.23)^{*}$ \\
\hline$\left[\begin{array}{ll}\mathrm{O} & \mathrm{I}\end{array}\right]$ & 6364 & $1.93(0.42)$ & $(7.07)$ & $\ldots *$ & $1.25(0.86) *$ & $3.49(1.56) *$ & $3.32(0.39)^{*}$ & $2.99(1.47)^{*}$ & $4.23(1.15) *$ \\
\hline$\left[\begin{array}{ll}\mathrm{Fe} & \mathrm{X}\end{array}\right]$ & 6375 & $24.6(2.98)$ & $6.13(1.59)^{*}$ & $28.6(4.06)^{*}$ & $11.6(1.22)^{*}$ & $5.96(1.97)^{*}$ & $6.83(1.13)^{*}$ & $8.38(0.87)^{*}$ & $7.14(1.26)^{*}$ \\
\hline He I & 6680 & $12.6(3.38)$ & $4.76(1.15)^{*}$ & $(1.33)$ & $3.85(0.38)^{*}$ & $2.44(1.49)^{*}$ & $2.53(0.25) *$ & $4.53(0.49)^{*}$ & $7.63(1.36)^{*}$ \\
\hline$\left[\begin{array}{ll}\mathrm{S} & \mathrm{II}\end{array}\right]$ & 6717 & (2.19) & $12.1(1.95)$ & $(1.26)$ & (1.18) & $1.82(0.08)$ & $2.22(0.55)$ & $1.62(0.22)^{*}$ & $1.43(0.19) *$ \\
\hline$[\mathrm{S}$ II $]$ & 6731 & $(1.33)$ & (1.79) & $(1.30)$ & $(0.86)$ & $1.61(0.26)$ & $2.31(0.56)$ & $1.30(0.13)$ & $1.53(0.14)^{*}$ \\
\hline He I (Nar.) & 7065 & $24.6(1.93)$ & $41.3(4.53)$ & $24.7(2.15)$ & $20.2(1.65)$ & $10.2(0.72)$ & $7.92(0.72)$ & $6.73(0.34)$ & $6.43(1.04)$ \\
\hline He I (Tot.) & 7065 & $24.6(1.93)$ & $41.3(4.53)$ & $39.1(2.63)$ & $35.6(1.69)$ & $21.3(0.91)$ & $29.8(1.44)$ & $29.9(2.67)$ & $30.6(2.24)$ \\
\hline [Ar III] & 7136 & (1.64) & $10.3(1.53)$ & $6.11(0.92)^{*}$ & $4.79(0.53)^{*}$ & $3.75(0.64)$ & $3.08(0.55)$ & $2.89(0.27)$ & $2.62(0.29)$ \\
\hline$\left[\begin{array}{ll}\mathrm{Fe} & \mathrm{II}\end{array}\right]$ & 7155 & $(1.95)$ & $(2.65)$ & $9.25(0.95) *$ & $9.71(1.33)^{*}$ & $12.5(0.80)$ & 10.4(1.61) & $6.91(0.56)$ & $5.67(0.65)$ \\
\hline He I & 7281 & $(2.50)$ & $(2.92)$ & $7.39(0.61) *$ & $(1.69)$ & $(1.57)$ & $1.17(0.29)^{*}$ & $(0.41)$ & $2.54(0.66)^{*}$ \\
\hline [O II] & 7325 & $(1.88)$ & $(4.23)$ & $13.5(3.49)^{*}$ & $(2.14)$ & $(2.61)$ & $4.00(0.34)$ & $1.98(0.25)^{*}$ & $1.89(0.15)^{*}$ \\
\hline$\left[\begin{array}{ll}\mathrm{S} & \mathrm{XII}\end{array}\right]$ & 7611 & $6.11(1.71)^{*}$ & $(1.60)$ & $5.12(0.38)$ & $(2.03)$ & $2.11(0.29)$ & $2.67(0.69)^{*}$ & $2.09(0.14)$ & $1.76(0.10)$ \\
\hline$[\mathrm{Fe}$ IV] $]$ & 7704 & $(0.82)$ & $(1.33)$ & $3.66(0.40)$ & $3.98(0.56)$ & $2.97(1.01)$ & $(0.29)$ & $(0.27)$ & $1.63(0.23)$ \\
\hline$\left[\begin{array}{ll}\mathrm{Fe} & \mathrm{xI}\end{array}\right]$ & 7891 & 13.4(1.34) & $(3.15)$ & $(1.37)$ & $3.61(0.33)$ & $4.73(0.73)$ & $5.12(0.74)$ & $4.63(0.60)$ & $4.80(0.23)$ \\
\hline He I, $\left[\begin{array}{ll}\mathrm{Fe} & \mathrm{VI}\end{array}\right]$ ? & 8232 & $1.92(0.57)^{*}$ & $(0.98)$ & $(1.80)$ & (1.01) & $2.86(1.00)$ & $2.89(0.48)$ & $7.48(2.05)^{*}$ & $7.55(0.56)^{*}$ \\
\hline He I & 8295 & $(1.74)$ & $(4.76)$ & $(3.20)$ & $2.51(0.20)^{*}$ & $3.68(0.65)$ & $1.99(0.36)$ & $2.37(0.54)$ & $2.06(0.43)$ \\
\hline $\mathrm{O}_{\mathrm{I}}$ & 8446 & $2.01(0.58)$ & $26.7(3.14)$ & $16.3(2.31)^{*}$ & $7.35(0.55)^{*}$ & $8.75(1.67)$ & $9.78(2.24)$ & $29.8(1.90)^{*}$ & $41.4(5.79)^{*}$ \\
\hline$[\mathrm{Fe} \quad \mathrm{VII}]$ & 8729 & $(1.66)$ & $15.4(3.51)^{*}$ & $(2.75)$ & $(0.64)$ & $(0.62)$ & $(0.39)$ & $(0.37)$ & $2.57(1.55)^{*}$ \\
\hline$[\mathrm{S}$ III $]$ & 9069 & $(2.14)$ & $22.8(1.51)$ & $14.9(1.76)$ & $10.5(1.22)$ & $11.3(2.12)$ & $4.84(1.07)^{*}$ & $\ldots$ & $5.13(1.01)^{*}$ \\
\hline
\end{tabular}

Note. - Fluxes are given in units of $10^{-16} \mathrm{erg} \mathrm{s}^{-1} \mathrm{~cm}^{-2}$. These are corrected for extinction and reddening of $E(B-V)=0.047 \mathrm{mag}$ in the Milky Way, but not for extinction local to SN 2005ip, which may be time dependent. Uncertainties for measured line fluxes are in parentheses; an upper limit is in parentheses when no measurement is given. Listed uncertainties are $1 \sigma$ based on the adjacent continuum noise, although the true uncertainties may be higher for blended lines, which are marked by *.

from the CSM interaction region, but detailed radiative transfer models are probably needed for a deeper understanding (e.g., Fransson et al. 2002).

In general, though, lines arising from a range of excitation levels and a range of densities are seen at all times, with neutral atoms up to high-ionization features like [Fe XIV]. One normally associates such a wide range of ionization with post-shock cooling zones, but as noted above, all these lines except $\mathrm{H} \alpha$ and He I are narrow lines emitted exclusively by as-yet unshocked CSM gas.
From this qualitative fact we infer that the photoionized pre-shock CSM of SN 2005ip must be clumpy or highly asymmetric, providing a target with a range of densities and optical depths within the same radial zone outside the forward shock. The various coronal lines also sample a wide range of critical densities up to $10^{7}-10^{8}$ $\mathrm{cm}^{-3}$, and this is presumably the reason why many lines are suppressed in the day 1 spectrum — in some cases transitions of the same ionization levels that are seen on day 1. For example, the low ratio of [Fe VII] $\lambda 5159 / \lambda 6086$ 
$\lesssim 0.05$ on day 1 implies electron densities around $10^{8}$ $\mathrm{cm}^{-3}$ or higher (Nussbaumer \& Storey 1982). Later, during the plateau phase, various [Fe VII] line ratios suggest similarly high densities and high gas temperatures above $10^{5} \mathrm{~K}$.

Although a range of densities is present at all times, it is likely that the average density falls at late times. In that case, one would infer that lines with lower critical densities that are collisionally de-excited at early times would "turn on" only at late times, when the shock and its photoionized precursor reach large radii where the wind density is sufficiently low. The clearest example of this effect is seen in the behavior of the [S II] $\lambda \lambda 6717$, 6731 and [O II] $\lambda 3726,3729$ doublets (Fig. 5] and Table $4)$; these lines are absent until the start of the late-time plateau phase when they both abruptly turn-on at day 173. Both transitions have critical densities of $n_{c} \approx 10^{4}$ $\mathrm{cm}^{-3}$. Much above that density, the lines are collisionally de-excited, suppressing the line intensity roughly proportional to $n_{c} / n_{e}$. Comparing the upper limits for [O II] on day 109 to the flux measured on day 173 then suggests minimum electron densities of $\gtrsim 3 \times 10^{5} \mathrm{~cm}^{-3}$ on day 109 and earlier. After that point, the [S II] ratio reflects densities of a few $10^{2} \mathrm{~cm}^{-3}$. It is likely that [S II] and [O II] trace lower densities between clumps, while the narrow coronal lines sample the densest regions in the clumps themselves. The clumpy nature of the progenitor wind is discussed further in $\S 4.1$.

In general, we can broadly categorize the observed evolution of line intensities in SN 2005ip into three main phases:

(1) In the initial state on day 1 , only a subset of narrow lines is seen, probably corresponding to those with the highest critical densities in the inner wind. The absence of any low-ionization features implies a very hard source of ionizing photons corresponding to soft X-rays rather than to the far ultraviolet. There is not yet any signature of strong CSM interaction in the day 1 spectrum (i.e., no intermediate-width components of $\mathrm{H} \alpha$ or He I), but these features may be hard to see at early times due to the high luminosity of the SN photosphere. If the X-rays required to drive the coronal lines were supplied by the X-rays arising from the initial shock breakout (e.g., Matzner \& McKee 1999; Ensman \& Burrows 1992), this would imply a progenitor radius much smaller than that of an extreme red supergiant (RSG) as we infer later (\$4.2).

(2) During the main decline of the SN light curve, lines either disappear and recover slowly or have a "hump" where they strengthen and then fade, as noted earlier. These changes likely reflect a combination transitional phenomenon, where the initial coronal lines on day 1 respond to the different recombination timescales appropriate to the range of ionization and densities in the clumpy inner wind. Simultaneously, as the forward shock expands, the pre-shock CSM density drops and new features arise, responding to both the dropping SN luminosity and the rising importance of luminosity generated by CSM interaction.

(3) During the plateau phase, nearly all narrow forbidden lines strengthen or remain steady (Fig. 12). This is true for all narrow lines except permitted lines like He I and $\mathrm{H} \alpha$ that are also seen in the dense post-shock gas. (Their narrow components fade at late times.) Sustain- ing the luminosity of coronal lines with such high ionization potentials for $\sim 3$ yr or more, and in some cases causing them to get stronger, requires a continued flux of soft $\mathrm{X}$-rays. Therefore, these lines cannot arise as a result of photoionization by X-rays generated during shock breakout alone. Instead, the photoionizing X-rays to drive the late-time coronal emission need to be generated in quasisteady state by CSM interaction. An X-ray luminosity of $1.6 \times 10^{40} \mathrm{erg} \mathrm{s}^{-1}$ was detected by Immler \& Pooley (2007) on day 466, consistent with this hypothesis.

\section{DISCUSSION}

\subsection{The Progenitor's Mass-Loss Rate}

We estimate the mass-loss rate of the progenitor star using two independent methods: the minimum mass in the CSM required to drain the necessary energy from the $\mathrm{SN}$, and the density of the CSM at a particular radius based on emission lines from the pre-shock CSM.

In order for CSM interaction to tap the kinetic energy reservoir of the expanding SN ejecta, the CSM must supply sufficient inertia. Thus, the observed bolometric luminosity, if attributable to CSM interaction alone, provides a minimum requirement for the mass-loss rate of the progenitor star. The required progenitor mass-loss rate (e.g., Chugai \& Danziger 1994) is typically given by

$$
\dot{M}=\frac{2 L}{\epsilon} \frac{V_{w}}{V_{S N}^{3}}
$$

where $\epsilon<1$ is the efficiency of converting shock kinetic energy into visual light (an uncertain quantity), $V_{w}$ is the progenitor's wind speed, $V_{S N}$ is the speed at which the CSM is overtaken by the SN, and $L$ is the observed CSM-interaction luminosity. At late times after day $\sim 170$, SN 2005ip shows a constant-luminosity plateau at $\log _{10}\left(L / \mathrm{L}_{\odot}\right)=41.45$, which we attribute to CSM interaction alone. With a pre-shock speed of $V_{w} \approx 120 \mathrm{~km}$ $\mathrm{s}^{-1}, V_{S N}=10^{4} \mathrm{~km} \mathrm{~s}^{-1}$, and $\epsilon \lesssim 0.5$ (an efficiency of $50 \%$ is probably the most optimistic value, although at lower optical depth the efficiency may be less; see Smith \& McCray 2007), we have

$$
\dot{M}=2.1 \times 10^{-4}\left(\frac{\epsilon}{0.5}\right)^{-1}\left(\frac{V_{w}}{120}\right)\left(\frac{V_{S N}}{10^{4}}\right)^{-3} \mathrm{M}_{\odot} \mathrm{yr}^{-1} .
$$

An independent way to estimate the progenitor's massloss rate is to infer values of the pre-shock wind density and speed at a specific radius. Earlier in $\S 3.7$ we estimated that the pre-shock wind density illuminated by SN 2005ip on day 109 was $\gtrsim 3 \times 10^{5} \mathrm{~cm}^{-3}$, based on the conjecture that the narrow $[\mathrm{O}$ II $]$ and $[\mathrm{S}$ II] doublets were suppressed until that date (they appeared in our next epoch of spectra on day 173) due to collisional de-excitation because of high densities in the pre-shock CSM. This minimum density represents the density of the interclump wind, occupying most of the wind volume; the much higher electron densities indicated by coronal lines correspond to the dense clumps with a small filling factor. On day 109, the BVZI of the broad $\mathrm{H} \alpha$ component was $\sim 16,900 \mathrm{~km} \mathrm{~s}^{-1}$ (Table 3 and Fig. 7). This speed is seen in the fast SN ejecta near the reverse shock, so the radius of the forward shock must be at least $R \gtrsim V t=1.6 \times 10^{16} \mathrm{~cm}$. At that radius, the inferred density places another lower limit to the pro- 
genitor's mass-loss rate, given by $\dot{M}=4 \pi R^{2} n_{e} m_{H} V_{w}$, of roughly $2.2 \times 10^{-4} \mathrm{M}_{\odot} \mathrm{yr}^{-1}$.

These two independent estimates give good agreement that the progenitor star's mass-loss rate was of order $\dot{M}=2 \times 10^{-4} \mathrm{M}_{\odot} \mathrm{yr}^{-1}$. There are considerable uncertainties involved, however. The estimates above were really lower limits to the required mass-loss rate because of efficiency and clumping, respectively, so we therefore adopt $\dot{M}=(2-4) \times 10^{-4} \mathrm{M}_{\odot} \mathrm{yr}^{-1}$ as a representative range of values for discussion here. This represents the progenitor's mass-loss rate for at least $300 \mathrm{yr}$ prior to core collapse. Also, both estimates are proportional to the inferred value of $V_{w}$. We adopted $V_{w} \approx 120 \mathrm{~km} \mathrm{~s}^{-1}$ because this corresponds to the marginally resolved width of the narrow-line components on day 413. As we noted earlier, however, the faster velocities indicated at earlier times hint that the pre-shock CSM could have been accelerated by the radiation force of the luminosity from the SN itself (e.g., Chugai et al. 2002), having a stronger effect at smaller radii probed at early times. Whether the progenitor wind was relatively fast $\left(120-150 \mathrm{~km} \mathrm{~s}^{-1}\right)$ or slow $\left(10-40 \mathrm{~km} \mathrm{~s}^{-1}\right)$ has important implications for the nature of the progenitor star (see Smith et al. 2007), but doesn't change many of the other conclusions we draw.

A more robust number is the wind density parameter $w=\dot{M} / V_{w}$ (making the derived result independent of $V_{w}$ ), which is equal to roughly $(1-2) \times 10^{15} \mathrm{~g} \mathrm{~cm}^{-1}$ for SN 2005ip. By day 905, the BVZI of $\mathrm{H} \alpha$ still indicates speeds of $\sim 14,000 \mathrm{~km} \mathrm{~s}^{-1}$ seen in the vicinity of the reverse shock. At that time, the minimum radius is then $\sim 10^{17} \mathrm{~cm}$. With the values above, the total CSM mass swept up by SN 2005ip on day 905 is at least $0.05-0.1 \mathrm{M}_{\odot}$ if the progenitor's mass-loss rate had been constant. SN 2005ip's CSM mass is considerably smaller than for many other SNe IIn, which have much higher values of $w \gtrsim 10^{16} \mathrm{~g} \mathrm{~cm}^{-1}$ and total masses that may be of order $0.5,1$, or even $10 \mathrm{M}_{\odot}$ (Chugai \& Danziger 1994; Smith et al. 2007, 2008b). This seems intuitively reasonable for SN 2005ip, however, and paints a self-consistent picture because SN 2005ip also had a relatively low CSMinteraction luminosity compared to other SNe IIn, and its low-mass CSM apparently failed to significantly decelerate the bulk of the fast SN ejecta.

\subsection{The Progenitor's Clumpy Wind}

If the CSM fails to decelerate the forward shock, then how can one explain the slower post-shock gas indicated by the persistent intermediate-width components of $\mathrm{H} \alpha$ and He I lines at late times? This problem is demonstrated in Figure 13. The intermediate-width lines are usually attributed to a cold dense shell that forms at the contact discontinuity between the forward shock and reverse shock (e.g., Chevalier 1982; Chugai 2001). As time proceeds, the fast SN ejecta that reach the reverse shock should have progressively lower speeds appropriate for the radius of the cold dense shell. In SN 2005ip, the minimum radius where material crosses the reverse shock advances at roughly $14,000 \mathrm{~km} \mathrm{~s}^{-1}$ (Fig. 13), which is much faster than the speed of the cold dense shell at $\sim 1000 \mathrm{~km} \mathrm{~s}^{-1}$. This cannot be.

A possible solution to this inconsistency is that the progenitor's wind is not homogeneous and spherical, but may be highly clumped or asymmetric, as proposed for

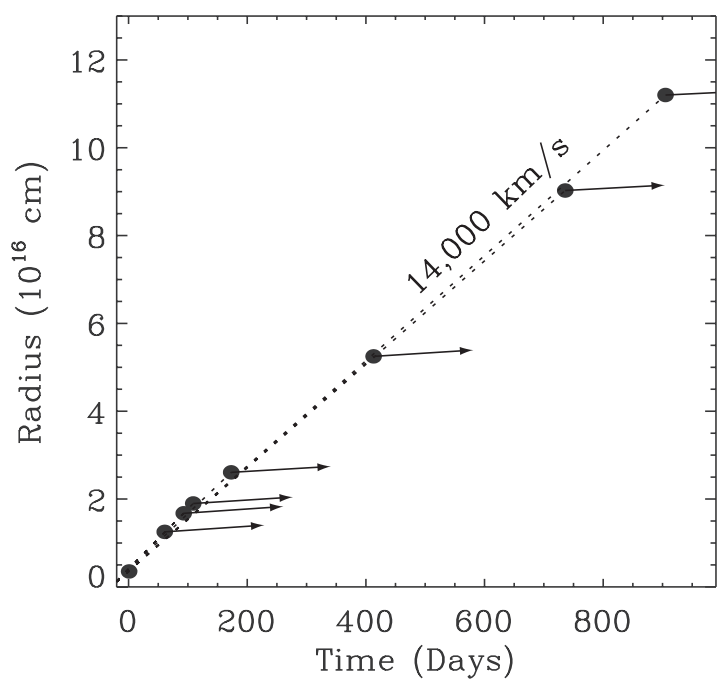

Fig. 13.- Filled circles show the minimum radius of the reverse shock in SN 2005ip as a function of time (indicated by the observed BVZI of $\mathrm{H} \alpha$ ), as well as the trajectories of the fast SN ejecta that are crossing the reverse shock at the observed epochs (dotted lines). These are plotted assuming that day 1 is actually $21 \mathrm{~d}$ after explosion, although this value is not well constrained (see text). The average expansion speed of the advancing reverse shock position is $\sim 14,000 \mathrm{~km} \mathrm{~s}^{-1}$. The solid arrows show the corresponding trajectories for slower gas giving rise to the intermediate-width components of emission lines.

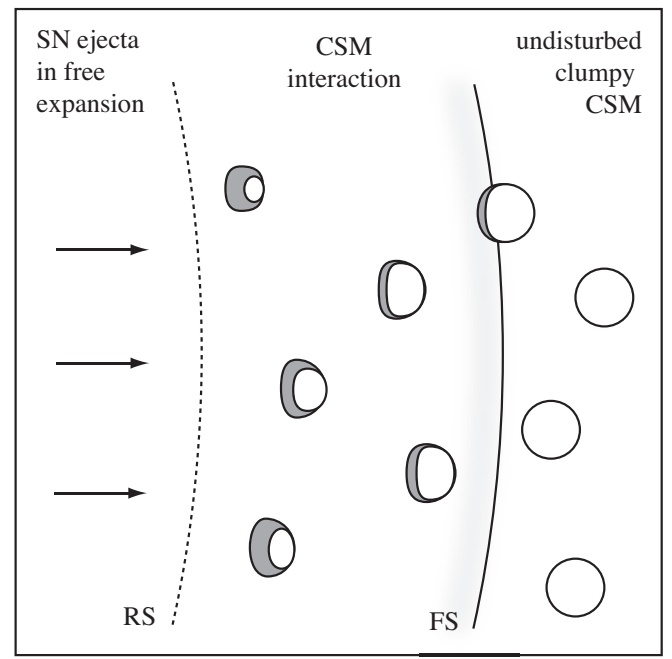

FIG. 14.- A sketch showing a possible interpretation of the CSMinteraction region in a clumpy wind around SN 2005ip, analogous to the model proposed for SN 1988 Z by Chugai \& Danziger (1994). A fast forward shock propagates into the CSM in the less-dense regions between clumps. As dense clumps in the CSM (white circles) are overtaken by the forward shock (solid curve), a slower shock is driven into each clump. This interaction destroys the clumps in the time it takes the shock to cross the clump. The dense post-shock gas in these clumps (gray) gives rise to the intermediate-width components of $\mathrm{H} \alpha$ and He I lines. X-rays from the more rarefied gas between the forward shock and reverse shock excite the narrow coronal lines in the unshocked regions of clumps on both sides of the forward shock. The geometry of the reverse shock is unclear, because reflected shocks propagating back into the SN ejecta may complicate the geometry. 
SN $1988 Z$ by Chugai \& Danziger (1994). An example situation corresponding to a clumpy wind is sketched in Figure 14. In this scenario, there is no coherent cold dense shell. Instead, the forward shock maintains a high speed, passing largely unhindered through the lower density regions between clumps. When the forward shock encounters a dense obstacle like a clump or a dense equatorial disk, a much slower shock is driven into the dense gas. The clumps in the CSM may penetrate well into the region engulfed by the expanding SN ejecta, surviving for a time corresponding to the cloud crushing time $t_{c} \approx a / V_{s}$, where $a$ is the characteristic radius of a clump and $V_{s}$ is the speed of the shock driven into the clump (about $1000 \mathrm{~km} \mathrm{~s}^{-1}$ ). With $a<<R$, we have $t_{c} \approx 100 \mathrm{~d}$. Thus, new generations of clumps in the outer wind are likely to be continually overtaken by the forward shock as older clumps are destroyed. Since $V_{s} \approx 0.1\left(V_{S N}\right)$, the clumps are likely to be about 10 times denser than the rarefied interclump wind.

The post-shock gas in the clumps (gray in Fig. 14) is denser still. We attribute the intermediate-width components of $\mathrm{H} \alpha$ and He I to emission from this gas. Extremely high densities above $10^{9} \mathrm{~cm}^{-3}$ are indicated by the high $\mathrm{H} \alpha / \mathrm{H} \beta$ ratio (Table 3 ) and the presence of these permitted lines combined with the lack of any intermediate-width component in any forbidden lines. High densities lead to efficient cooling, suppressing the coronal lines in the post-shock gas and potentially allowing the gas to cool enough to form dust. Similar densities around $10^{10} \mathrm{~cm}^{-3}$ were inferred in the post-shock dustforming regions of SN 2006jc (Smith et al. 2008a).

Small-scale clumps embedded in an otherwise spherical wind with mass-loss rates of the order required for SN 2005ip are reasonable and expected from studies of evolved stars. In fact, small-scale clumps have now been directly detected in the CSM of the extreme RSG star VY CMa out to $10^{17} \mathrm{~cm}$ around the star (Smith, Hinkle, \& Ryde 2009) - this is the identical range of radii swept up by SN 2005ip. Smith et al. (2009) also showed that the mass-loss rate of VY CMa, $(2-4) \times 10^{-4} \mathrm{M}_{\odot} \mathrm{yr}^{-1}$, and other conditions are suitable to the production of a Type IIn event like SNe 2005ip or 1988Z. Fransson et al. (2002) made a similar comparison to VY CMa for SN 1995N. If this link to VY CMa holds true, then it suggests that SNe like 1988Z, 1995N, and 2005ip arise from fairly rare and extreme RSGs that had initial masses of roughly 20 $40 \mathrm{M}_{\odot}$. Chugai \& Danziger (1994) have suggested that a slight depression in the intermediate-width $\mathrm{H} \alpha$ component may signal an equatorial concentration as opposed to clumps; we see no sign of this effect except for a very slight central depression on day 736. Also, these authors commented that the signature of large-scale clumps may be seen in irregular structure of the intermediatewidth component's profile. We see no sign of this irregular structure either, consistent with our claim that the clumps in question are an ensemble of small clumps, distributed roughly symmetrically throughout the wind.

\subsection{Dust Formation in Both the Ejecta and Shell}

Recently, Fox et al. (2008) reported that SN 2005ip had a strong near-IR excess over a time period of more than $900 \mathrm{~d}$. The $J H K$ fluxes were consistent with hot dust at $1400-1600 \mathrm{~K}$. Based on the similar behavior of SN 2006jc (Smith et al. 2008a) and other considerations,
Fox et al. showed that the IR excess of SN 2005ip was consistent with dust forming in the swept-up cold dense shell in the post-shock layer, rather than an IR echo.

An unambiguous sign of the formation of new dust grains in a SN is when one sees an increasing amount of extinction in the SN line profiles. Specifically, as an increasing amount of dust forms, it should preferentially block more of the redshifted side of the ejecta. This leads to a systematic fading of the red sides of emission lines and a net blueshift of the line centroid. So far, though, this effect has only been clearly documented in a few cases, like SN 1987A (Danziger et al. 1989; Lucy et al. 1989; Gehrz \& Ney 1989; Wooden et al. 1993; Colgan et al. 1994; Wang et al. 1996; Moseley et al. 1989; Dwek et al. 1992), SN $1999 \mathrm{em}$ (Elmhamdi et al. 2003), and SN 2003gd (Sugerman et al. 2006; Meikle et al. 2007). In all three, the dust formed in the fast SN ejecta.

A second possibility is that in rare cases of $\mathrm{SNe}$ with strong CSM interaction, dust grains may also form in the dense post-shock cooling shell. The strongest case for this type of dust formation was SN 2006jc, which showed the characteristic blueshift in its intermediatewidth He I line profiles, and simultaneously increased continuum extinction and IR excess emission from hot dust (Smith et al. 2008a). ${ }^{3}$ Smith et al. speculated that this post-shock dust formation occurred in SN 2006jc a peculiar Type Ib or Ibn - because the forward shock encountered a dense CSM shell ejected 2 yr before, decelerating the shock and forming a dense $\left(\sim 10^{10} \mathrm{~cm}^{-3}\right)$ post-shock layer that could cool fast enough to form dust. Smith et al. conjectured that it may be easier to form this dust in decelerated C-rich ejecta that have passed the reverse shock, although this was difficult to prove from observations. Post-shock dust formation may also have been seen in the Type IIn events SN 1998S (Pozzo et al. 2004) and SN 2006tf (Smith et al. 2008b). In hindsight, dust formation in the post-shock gas may also have caused the fading of the red wings of intermediate-width $\mathrm{H} \alpha$ components in SN $1995 \mathrm{~N}$ noted by Fransson et al. (2002). This suggests that this mode of dust formation may be more generic and may not require C-enriched material.

As noted above in $§ 3.6$, SN 2005ip shows evidence for dust formation in the post-shock shell based on the behavior of intermediate-width He I line profiles (Fig. 8). This, in principle, confirms the recent suggestion by Fox et al. (2008).

There are two caveats we add, however. First, suppression of the red wings of the He I lines in SN 2005ip is only seen at late times, more than $1 \mathrm{yr}$ after explosion and later, whereas Fox et al. (2008) reported a strong near-IR excess present much sooner after explosion during the main decline in the light curve. Thus, while we do see evidence for dust formation in the post-shock gas at a time when an IR excess is present, the IR excess was already present and did not increase substantially when evidence for new dust developed in the He I line profiles. Therefore, we cannot confirm that the IR excess before day 736 was due to post-shock dust formation, as op-

3 Although Nizawa et al. (2008) proposed that dust formed early in the ejecta of SN 2006jc, both Smith et al. (2008a) and Matilla et al. (2008) had found that dust forming in the fast SN ejecta could not account for the He I line-profile evolution, and concluded that the dust must form in the post-shock shell. 
posed to it having some other possible origin. Second, as noted in $§ 3.6$, we also see strong evidence for new dust formation occurring in the fast SN ejecta based on the behavior of the broad $\mathrm{H} \alpha$ profile - but it formed much sooner after the explosion, during the main decline of the SN light curve. Coincidentally, this is also when the IR excess from hot dust was first seen by Fox et al. (2008).

Thus, from the line-profile evolution of SN 2005ip we conclude that the IR excess from hot dust reported by Fox et al. (2008) can arise from new dust grains in either the fast SN ejecta or the post-shock shell, or perhaps a combination of both at different times. There seem to have been two episodes of dust formation: (1) dust formed first in the rapidly expanding SN ejecta at days 60-170, causing the initial near-IR excess seen at those times and affecting the broad $\mathrm{H} \alpha$ line profile, and (2) dust also formed much later in the post-shock shell, sustaining the IR plateau at late times and shaping the He I lines. The luminosity source that re-heats the dust in both zones may be generated by CSM interaction (propagating inward to the dusty SN ejecta and outward to the cold dense post-shock gas). This is consistent with the observation that the late-time visual and IR luminosities are both $\sim 10^{41.5} \mathrm{~L}_{\odot}$.

SN 2005ip provides a unique case among known SNe in that it shows strong evidence for dust formation in both the fast SN ejecta and also in the slow and dense post-shock gas. So far, the SNe IIn 2005ip, 1998S (Pozzo et al. 2004), and 2006tf (Smith et al. 2008b), as well as the SN Ibn 2006jc (Smith et al. 2008a), all seem to have formed dust in their swept-up shells. This hints that post-shock dust formation may be common in SNe with strong CSM interaction, and implies that we may need to revisit the cause of the IR excess emission in SNe IIn that has usually been interpreted as light echoes, as noted also by Fox et al. (2008). This difference is important because dust that forms in fast SN ejecta or pre-existing dust in the CSM is destined to be destroyed when it passes the reverse shock or forward shock, respectively. Dust forming in the cold dense shell, however, is already in the post-shock layer and will likely survive.

In previous examples of SNe where dust formed in the post-shock gas, it was assumed that this occurred in the cold dense shell between the forward and reverse shocks, as in SN 2006jc (Smith et al. 2008a) and SN 1998S (Pozzo et al. 2004). In the clumpy model suggested here, SN 2005ip does not have a coherent cold dense shell, so where does the dust form in a scenario like that depicted in Figure 14]? In order to block the intermediate-width components at late times, the dust may form in either the dense gas behind the shocks driven into dense clumps or in the SN ejecta themselves. As we noted earlier, however, evidence for extinction from new dust is not seen in the intermediate-width He I profiles until late times (Fig. 8). An intriguing possibility that may reconcile the IR analysis by Fox et al. (2008) and the line-profile evolution that we observe may be that dust forms in the dense post-shock gas within individual clumps, but then gets ablated by and incorporated into the expanding fast SN ejecta when a clump is eventually destroyed. This may provide an explanation for the dust present in the SN ejecta that are otherwise presumably still too hot to form dust at 60-170 d after explosion. The radius we infer at days $60-170$ when dust appeared is around $10^{16} \mathrm{~cm}$
(Fig. 13), consistent with the minimum radius expected from the study of SN 2005ip's IR emission by Fox et al. (2008). A more detailed analysis of this interaction is beyond the scope of this work, but it is apparently consistent with the evidence we see for dust forming in the fast SN ejecta as indicated by the evolution of the broad $\mathrm{H} \alpha$ profile.

This is also the first report of both post-shock dust formation and strong coronal line emission in a SN, each linked to CSM interaction, although Smith et al. (2008a) noted the similar coincidence of dust formation and He II $\lambda 4686$ emission in SN 2006jc and $\eta$ Carinae. Classical novae also show signs of dust formation and coronal emission, but these two are rarely seen together in the same object (Gehrz et al. 1998). The combination of both dust formation and coronal emission has been reported only a few times in classical novae (e.g., Gehrz et al. 1995, 2008; Mason et al. 1996 and references therein).

\subsection{CSM Interaction in Context: A Comparison with SNe 1988Z, 2006jc, and Others}

The most concise way to summarize the behavior of SN 2005ip is that it is nearly a carbon copy of SN 1988Z, although less luminous (Stathakis \& Sadler 1991; Turatto et al. 1993; Chugai \& Danziger 1994). It is likely that SN $1995 \mathrm{~N}$ is closely related as well (Fransson et al. 2002), but a comparison of the time evolution is difficult because SN 1995N was discovered late. The most remarkable similarities between SNe 2005ip and 1988Z are their nearly identical $\mathrm{H} \alpha$ profiles with three components (broad, intermediate-width, and narrow), the way their velocities evolve with time, the parabolic shape of the broad $\mathrm{H} \alpha$ component, the lack of P Cygni absorption (except in the broad component at day 1 in SN 2005ip), and the very high (well above 10$) \mathrm{H} \alpha / \mathrm{H} \beta$ ratio. Given these similarities, we would predict that SN 2005ip should be an extremely luminous radio source for years after explosion, akin to SN 1988Z (Van Dyk et al. 1993; Williams et al. 2002) and SN 1995N (Chandra et al. 2008). The close comparison with SN 1988 Z holds with the few following caveats:

(1) SN 2005ip had a lower visual luminosity, both in its initial decline and during its late-time luminosity plateau powered by CSM interaction. At 1 yr after explosion it was about $2 \mathrm{mag}$ fainter, while after $2.5 \mathrm{yr}$ this difference had reduced so that SN 2005ip was only 0.5 mag fainter in visual light.

(2) Despite its lower visual luminosity, the X-ray luminosity of SN 2005ip observed by Immler \& Pooley (2008) was comparable to that of SN 1988Z, so SN 2005ip has a relatively high value of $L_{X} / L_{B o l}$.

(3) The fastest speeds seen in the broad $\mathrm{H} \alpha$ component were similar in both SNe at early times, $(19-20) \times 10^{3} \mathrm{~km}$ $\mathrm{s}^{-1}$, but the speeds did not drop as much in SN 2005ip at late times.

(4) Although SN 1988Z did show some coronal lines, the coronal spectrum in SN 2005ip was much more prominent and the coronal lines were more numerous. The coronal lines dominated the spectrum in way that has not been seen in any previous SN, indicating pervasive hot gas in the CSM. The blue pseudo-continuum, composed of many blended narrow lines, was also more prominent in SN 2005ip. 
We suggest that all four of these differences compared to SN 1988 Z can be explained by the hypothesis that SN 2005ip's progenitor had a lower mass-loss rate. The wind density parameter we derive for SN 2005ip's clumpy wind is about 5-10 times lower than that which Chugai \& Danziger (1994) derived for SN 1988Z. The lower wind density is less able to decelerate the fast SN ejecta, explaining the sustained higher speeds, and is therefore less able to drain kinetic energy from the fast ejecta, resulting in a lower luminosity. The lower density also provides for a lower efficiency in converting $\mathrm{X}$-rays to visual light because of the lower optical depth within the post-shock layers. Consequently, since it is less able to absorb the X-rays (and perhaps also by producing a harder radiation field via the faster shock speed), SN 2005ip generates a relatively stronger radiative X-ray precursor that illuminates the pre-shock CSM and drives the coronal spectrum.

We also noted some interesting similarities between SN 2005ip and SN 2006jc. The He I line profiles in SN 2006jc are similar to those in the later stages of SN 2005ip. Both objects also show evidence for postshock dust formation, plus a pronounced blue continuum and flat red continuum that cannot be fit with any blackbody. By virtue of the narrow CSM lines in SN 2005ip, we see for the first time that this blue pseudo-continuum (also present in SN 1988Z to a lesser degree) is, in fact, composed of a large number of blended emission lines originating in the CSM. We had suspected this in the case of SN 2006jc (Foley et al. 2007). The blue continuum is probably fluorescence of dense CSM gas illuminated by a strong radiative precursor with a fairly hard spectrum, as in AGNs, although a quantitative study of the origin of this emission remains to be conducted. Since SN 2005ip had H-rich CSM, this connection proves that the similar blue continuum in SN 2006jc was not a result of low opacities in H-depleted gas.

\subsection{Why Aren't There More SNe Like SN 2005ip?}

In many ways, SN 2005ip is what one might think that most SNe IIn should look like. The underlying SN was not extraordinarily luminous, nor was the ongoing CSM interaction luminosity very high, and the progenitor's clumped wind with a modest mass-loss rate of order $10^{-4}$ $\mathrm{M}_{\odot} \mathrm{yr}^{-1}$ is not unusual among known examples of luminous evolved massive stars. The steady late-time plateau suggests that this mass-loss rate was constant for centuries before core collapse, consistent with the relatively steady winds seen in the majority of massive stars. Why, then, have we not seen a SN IIn quite like SN 2005ip before, with a steady late-time plateau and such prominent coronal emission?

On the one hand, SN 2005ip had a relatively modest luminosity compared with other more luminous SNe IIn, and its late-time plateau was rather dim at only -14.8 mag $\left(\right.$ or $\log _{10}\left[L / \mathrm{L}_{\odot}\right]=41.45$ ). It was also relatively nearby at a distance of only $\sim 30 \mathrm{Mpc}$ and its host galaxy is faint, so it is reasonable to assume that there may be more SNe IIn with faint late-time plateaus like SN 2005ip that go undetected at larger distances. Many SNe IIn do, however, fade more than SN 2005ip in the few years after explosion (Li et al. 2002).

On the other hand, its peak luminosity did exceed that of a normal SN II-P, and a subset of the narrow coronal lines were in fact prominent in the day 1 spectrum near maximum light. Few SNe exhibit these coronal lines, and in those that do, the coronal spectrum is nowhere near as prominent as in SN 2005ip. This suggests that the origin of the coronal spectrum in SN 2005ip is the result of special circumstances.

We speculate, therefore, that SN 2005ip sits near a critical transition between the two regimes of normal SNe II and SNe IIn. The transition is defined by the progenitor star's mass-loss rate, such that SN 2005ip probably marks the lower bound of wind density where the wind is sufficiently dense to give rise to a Type IIn spectrum, but not so dense that the resulting CSM interaction becomes very optically thick. Furthermore, the clumpy nature of the wind acts, in effect, as a type of porosity that simultaneously provides dense gas while allowing the fast blast wave and X-rays to escape through it nearly unabated. This seems to be an essential ingredient to producing the coronal spectrum.

At higher progenitor mass-loss rates, the swept-up CSM would be more optically thick, leading to higher efficiency in reprocessing shock kinetic energy (and Xrays) into visual light as in SNe 2006tf, 2006gy, 2008es, and 2005ap (Smith et al. 2008b, 2007; Miller et al. 2008; Quimby et al. 2007), but simultaneously preventing the escaping X-ray flux that gives rise to the coronal spectrum in the pre-shock CSM. In the most luminous SNe IIn with higher progenitor mass-loss rates resulting from episodic pre-SN ejections (e.g., Smith \& Owocki 2006), the pre-SN mass loss is much higher than can be produced by a normal stellar wind. Thus, it is not clear that one expects those more extreme CSM environments to have the same degree of clumpiness, and the clumps might not survive in the optically thick post-shock region where the shock kinetic energy is efficiently thermalized.

Altogether, given the moderately high progenitor mass-loss rate of $(2-4) \times 10^{-4} \mathrm{M}_{\odot} \mathrm{yr}^{-1}$ in a clumpy wind, consistent with an extreme RSG like VY CMa (Smith et al. 2009), we suggest that the progenitor of SN 2005ip was a moderately massive star of roughly $20-40 \mathrm{M}_{\odot}$. It probably exploded at the apex of its RSG phase, when stars at that phase show the most vigorous mass loss and the densest CSM environments (Davies et al. 2008). In context with other SNe IIn, SN 2005ip's relatively modest luminosity and presumed origin from a moderately massive RSG progenitor underscore the much more extreme requirements for the luminous SNe IIn like SN 2006gy (Smith et al. 2007). Namely, it seems consistent with the idea that these even rarer and more extreme events arise from the explosions of the most massive stars, requiring episodic or luminous blue variable (LBV)-like mass ejections in their pre-SN phases (Smith et al. 2007). SN 2005ip's progenitor was probably also less massive than that of the SN IIn 2005gl, which GalYam et al. (2007) found to be consistent with a very massive LBV-like star.

\section{CONCLUSIONS}

We present and analyze visual-wavelength photometry and spectroscopy of SN 2005ip obtained at the Lick and Keck Observatories during a period of $\sim 3$ yr after explosion. Our main conclusions are as follows.

(1) The data show that SN 2005ip was composed of an underlying broad-lined Type II-L event that dominated 
the luminosity decline during the first $\sim 160 \mathrm{~d}$, superposed with a Type IIn spectrum arising from constantluminosity CSM interaction that caused a remarkably steady late-time plateau. SN 2005ip was not unusually luminous compared with other SNe IIn, and its initial mass of ${ }^{56} \mathrm{Ni}$ was less than about $0.1 \mathrm{M}_{\odot}$.

(2) As the underlying SN II-L faded, the spectrum of SN 2005ip came to exhibit a forest of narrow coronal emission lines, the number and strength of which dominated the spectrum to an unprecedented degree. Ionization levels as high as [Fe XIV] are seen. These forbidden coronal lines arise exclusively in the pre-shock CSM, showing no broader components from the post-shock gas or SN ejecta. Only a subset of the narrow coronal lines was present in the day 1 spectrum.

(3) Following the discovery by Fox et al. (2008) that SN 2005ip showed near-IR excess emission attributable to freshly synthesized dust, we confirm evidence for this dust formation from its influence on the evolution of visual-wavelength emission lines. Components of both He I $\lambda 7065$ and $\mathrm{H} \alpha$ have red wings that fade faster than the constant blue wings, as expected if new dust grains preferentially block the far side of the object. However, these two tracers reveal dust at different places at different times as the $\mathrm{SN}$ evolves. At very late times $(2-3 \mathrm{yr}$ after explosion), intermediate-width components of He I lines reveal dust forming in a post-shock shell analogous to the post-shock dust formation in SN 2006jc (Smith et al. 2008a), as proposed by Fox et al. (2008). At early times, however, the situation is qualitatively different: we see no evidence for dust in the intermediate-width components of these lines, but instead, we see the same fading of the red wing in the broad component of $\mathrm{H} \alpha$. This means that at early times, either (a) the dust formed directly in the fast SN ejecta, or (b) the dust formed in the post-shock gas of individual clumps, but was quickly incorporated into the rapidly expanding SN ejecta as the parent clumps were ablated and destroyed. At both epochs the dust in both locations is heated primarily by the constant luminosity from CSM interaction.

(4) The photometric and spectroscopic evolution of SN 2005ip was most similar to that of the strongly interacting and X-ray/radio-bright SN IIn 1988Z, especially with regard to the evolution of its $\mathrm{H} \alpha$ profile. The close comparison holds with the exceptions that SN 2005ip was less luminous, had a higher $L_{X} / L_{B o l}$ ratio than SN 1988Z, and exhibited stronger narrow coronal lines; moreover, the fastest speeds seen in SN 2005ip persisted longer than in SN 1988Z.

(5) We propose a model for SN 2005ip that is simi- lar to that of Chugai \& Danziger (1994) for SN 1988Z, wherein a SN II plows into a steady but clumpy progenitor wind. Dense clumps in the CSM persist into the postshock zone as they are overtaken by the forward shock passing through the low-density region between them, while much slower shocks are driven into individual dense clumps giving rise to the intermediate-width components of the $\mathrm{H} \alpha$ and $\mathrm{He}$ I lines. In this model, the differences between SNe 2005ip and 1988Z noted in point (4) above can be explained if the progenitor of SN 2005ip had a mass-loss rate a factor of $\sim 5$ lower than that of SN 1988Z, making its CSM interaction region less massive and less optically thick. The lower optical depth allows X-rays generated in the CSM interaction to thoroughly ionize unshocked CSM gas, giving rise to the coronal spectrum.

(6) Given the rarity of coronal spectra like that of SN 2005ip, and in light of the similarity of its inferred progenitor's wind to the extreme conditions observed in the winds of the most luminous known RSGs such as VY CMa, the most straightforward interpretation seems to be that moderate-luminosity SNe IIn like SN 2005ip arise from extreme RSGs having relatively high initial masses of 20-40 $\mathrm{M}_{\odot}$. Since the fairly modest luminosity of SN 2005ip is apparently near the limit of what can be achieved by interaction with the densest steady stellar winds known, this finding underscores the requirement that exceptionally luminous SNe IIn such as SNe 2006tf and 2006gy require much more extreme pre-SN mass ejections analogous to massive LBV eruptions (Smith et al. 2007, 2008b). At the same time, SN 2005ip demonstrates that not necessarily all core-collapse SNe IIn require this type of LBV-like mass ejection.

We acknowledge interesting discussions concerning the nature of SN 2005ip with O. Fox and R. Chevalier. Some of the data presented herein were obtained at the W.M. Keck Observatory, which is operated as a scientific partnership among the California Institute of Technology, the University of California, and the National Aeronautics and Space Administration (NASA). The Observatory was made possible by the generous financial support of the W.M. Keck Foundation. We wish to extend special thanks to those of Hawaiian ancestry on whose sacred mountain we are privileged to be guests. We are grateful to the staffs at the Lick and Keck Observatories for their dedicated services. Also, we acknowledge S. Park, D. Pooley, D. Poznanski, and D.S. Wong for assistance with some of the observations. KAIT was constructed and supported by donations from Sun Microsystems, Inc., the Hewlett-Packard Company, AutoScope Corporation, Lick Observatory, the US National Science Foundation (NSF), the University of California, the Sylvia \& Jim Katzman Foundation, and the TABASGO Foundation. A.V.F.'s supernova group at U.C. Berkeley is supported by NSF grant AST-0607485 and by the TABASGO Foundation.

\section{REFERENCES}

Aretxaga, I., et al. 1999, MNRAS, 309, 343

Arnett, D. 1996, Supernovae and Nucleosynthesis (New Jersey: Princeton University Press)

Boles, T. 2005, CBET, 275, 1

Chandra, P., et al. 2008, ApJ, submitted (arXiv:0809.2810)

Chevalier, R. A. 1982, ApJ, 259, 302

Chevalier, R. A., \& Fransson, C. 1994, ApJ, 420, 268

Chugai, N. N. 2001, MNRAS, 326, 1448

Chugai, N. N., \& Danziger, I. J. 1994, MNRAS, 268, 173

Chugai, N. N., Blinnikov, S.I., Fassia, A., Lundqvist, P., Meikle, W. P. S., \& Sorokina, E. I. 2002, MNRAS, 330, 473

Colgan, S. W. J., Haas, M. R., Erickson, E. F., Lord. S. D., \& Hollenbach, D. J. 1994, ApJ, 427, 874

Danziger, I. J., Gouiffes, C., Bouchet, P., \& Lucy, L. B. 1989, IAU Circ., 4796, 1
Davies, B., Figer, D. F., Law, C. J., Kudritzki, R. P., Najarro, F., Herrero, A., \& MacKenty, J. W. 2008, ApJ, 676, 1016

de Vaucouleurs, G., et al. 1991, Third Reference Catalogue of Bright Galaxies, ver. 3.9

Doggett, J. B., \& Branch, D. 1985, AJ, 90, 2303

Dwek, E., Moseley, S. H., Glaccum, W., Graham, J. R., Loewenstein, R. F., Silverberg, R. F., \& Smith, R. K. 1992, ApJ, 389, L21

Elmhamdi, A., Danziger, I. J., Chugai, N., Pastorello, A., Turatto, M., Cappellaro, E., Altavilla, G., Benetti, S., Patat, F., \& Salvo, M. 2003, MNRAS, 338, 939

Ensman, L., \& Burrows, A. 1992, ApJ, 393, 742

Faber, S. M., et al. 2003, Proc. SPIE, 4841, 1657

Filippenko, A. V. 1982, PASP, 94, 715

Filippenko, A. V., \& Sargent, W. L. W. 1989, ApJ, 342, L11 
Filippenko, A. V. 1991, in SN 1987A and Other Supernovae, ed. I. J. Danziger \& K. Kjär (Garching: ESO), 343

Filippenko, A.V. 1997, ARAA, 35, 309

Filippenko, A. V. 2005, in The Fate of the Most Massive Stars, ed. R Humphreys \& K. Stanek (San Francisco: ASP), 33

Filippenko, A. V., Li, W. D., Treffers, R. R., \& Modjaz, M. 2001, in Small-Telescope Astronomy on Global Scales, ed. W. P. Chen, C Lemme, \& B. Paczyński (San Francisco: ASP, Conf. Ser. Vol. 246) 121

Foley, R. J., Smith, N., Ganeshalingam, M., Li, W., Chornock, R., \& Filippenko, A. V. 2007, ApJ, 657, L105

Foley, R. J., et al. 2003, PASP, 115, 1220

Fox, O., et al. 2008, preprint (arXiv:0807.3555

Fransson, C., \& Gröningsson, P. 2007, in Supernova 1987A: 20 Years After, ed. S. Immler, K. Weiler, \& R. McCray (New York: AIP), 155

Fransson, C., et al. 2002, ApJ, 572, 350

Gal-Yam, A., et al. 2007, ApJ, 656, 372

Garnavich, P., \& Ann, H.B. 1994, AJ, 108, 1002

Gehrz, R. D., \& Ney, E. P. 1989, Proc. Nat. Acad. Sci., 87, 4354

Gehrz, R. D., Truran, J. W., Williams, R. E., \& Starrfield, S. 1998, PASP, 110, 3

Gehrz, R. D., Jones, T. J., Matthews, K., Neugebauer, G., Woodward, C. E., Hayward, T. L., \& Greenhouse, M. A. 1995, AJ, 110, 325

Gehrz, R. D., et al. 2008, ApJ, 672, 1167

Gordon, C. 1972, A\&A, 20, 79

Gröningsson, P., Fransson, C., Lundqvist, P., Nymark, T., Lundqvist, N., Chevalier, R., Leibundgut, B., \& Spyromilio, J. 2006, A\&A, 456, 581

Gröningsson, P., Fransson, C., Lundqvist, P., Lundqvist, N., Leibundgut, B., Spyromilio, J., Chevalier, Gilmozzi, R., Kjaer, K. Matilla, S., \& Sollerman, J. 2008, A\&A, 479, 761

Hoffman, J. L., Leonard, D. C., Chornock, R., Filippenko, A. V., Barth A. J., \& Matheson, T. 2008, ApJ, accepted, astro-ph/0709.3258

Immler, S., \& Pooley, D. 2007, ATel, 1004, 1

Leibundgut, B., Kirshner, R. P., Pinto, P. A., Rupen, M. P., Smith, R. C., Gunn, J. E., \& Schneider, D. P. 1991, ApJ, 372, 531

Li, W., et al. 2002, PASP, 114, 403

Li, W., Filippenko, A. V., Chornock, R., \& Jha, S. 2003, ApJ, 586, L9

Lucy, L. B., Danziger, I. J., Gouiffes, G., \& Bouchet, P. 1989, in Structure and Dynamics of the Interstellar Medium, ed. G. TenorioTagle, et al. (Berlin: Springer-Verlag), 164

Mason, C., Gehrz, R. D., Woodward, C. E., Smilowitz, J. B., Greenhouse, M. A., Hayward, T. L., \& Houck, J. R. 1996, ApJ, 470,577

Matilla, S., et al. 2008, MNRAS, 389, 141

Matzner, C. D., \& McKee, C. F. 1999, ApJ, 510, 379
Meikle, W. P. S., et al. 2007, ApJ, 665, 608

Miller, J. S., \& Stone, R. P. S. 1993, Lick Obs. Tech. Rep. 66

Miller, A., et al. 2008, ApJ, in press (arXiv:0808.2193

Modjaz, M., Kirshner, R., \& Challis, P. 2005, CBET, 276, 1

Monet, D.G., et al. 2003, AJ, 125, 984

Moseley, S. H., Dwek, E., Glaccum, W., Graham, J. R., Loewenstein,

R. F., \& Silverberg, R. F. 1989, Nature, 340, 697

Munari, U., \& Zwitter, T. 1997, A\&A, 318, 269

Nozawa, T., et al. 2008, ApJ, in press arXiv:0801.2015

Nussbaumer, H., \& Storey, P.J. 1982, A\&A, 113, 21

Oke, J. B., et al. 1995, PASP, 107, 375

Pastorello, A., et al. 2008, MNRAS, 389, 131

Pozzo, M., et al. 2004, MNRAS, 352, 457

Quimby, R. M., Aldering, G., Wheeler, J. C., Höflich, P., Akerlof, C. W., \& Rykoff, E. S. 2007, ApJ, 668, L99

Schlegel, D. J., Finkbeiner, D. P., \& Davis, M. 1998, ApJ, 500, 525

Smith, N., \& McCray, R. 2007, ApJ, 671, L17

Smith, N., \& Owocki, S. P. 2006, ApJ, 645, L45

Smith, N., Foley, R. J., \& Filippenko, A. V. 2008a, ApJ, 680, 568

Smith, N., et al. 2008b, ApJ, 686, 467

Smith, N., Hinkle, K. H., \& Ryde, N. 2009, AJ, in press (arXiv:0811.3037)

Smith, N., Li, W., Foley, R. J., Wheeler, J. C., Pooley, D., Chornock, R., Filippenko, A. V., Silverman, J. M., Quimby, R., Bloom, J., \& Hansen, C. 2007, ApJ, 666, 1116

Stathakis, R. A., \& Sadler, E. M. 1991, MNRAS, 250, 786

Sugerman, B. E. K., et al. 2006, Science, 313, 196

Thompson, T. A., et al. 2008, preprint, arXiv:0809.0510

Turatto, M., et al. 1993, MNRAS, 262, 128

Van Dyk, S. D. 2005, in The Fate of the Most Massive Stars, ed. R. Humphreys \& K. Stanek (San Francisco: ASP), 47

Van Dyk, S. D., Weiler, K.W., Sramek, R.A., \& Panagia, N. 1993, ApJ, 419, L69

Wagner, W. J., \& House, L. L. 1968, Solar Physics, 5, 55

Wang, L., Wheeler, J. C., Kirshner, R. P., Challis, P. M., Filippenko, A. V., Fransson, C., Panagia, N., Phillips, M. M., \& Suntzeff, N. 1996, ApJ, 466, 998

Williams, R. E., Hamuy, M., Phillips, M. M., Heathcote, S. R., Wells, L., \& Navarrete, M. 1991, ApJ, 376, 721

Williams, R. E., Panagia, N., Van Dyk, S. D., Lacey, C.K., Weiler, K.W., \& Sramek, R.A. 2002, ApJ, 581, 396

Wooden, D. H., Rank, D. M., Bregman, J. D., Witteborn, F. C., Tielens, A. G. G. M., Cohen, M., Pinto, P. A., \& Axelrod, T. S. 1993, ApJS, 88, 477

Woodward, C. E., et al. 1995, ApJ, 438, 921 